\title{
Core turbulence behavior moving from ITG towards TEM regime in the ASDEX Upgrade tokamak and comparison with gyrokinetic simulation
}

\author{
T. Happel, ${ }^{*}$ A. Bañón Navarro, G. D. Conway, C. Angioni, M. Bernert, M. Dunne, E. Fable, \\ B. Geiger, T. Görler, F. Jenko, R. M. McDermott, F. Ryter, U. Stroth, and the ASDEX Upgrade Team \\ Max Planck Institute for Plasma Physics, Boltzmannstr. 2, 85748 Garching, Germany
}

(Dated: 2017-01-26)

\begin{abstract}
Additional electron cyclotron resonance heating $(\mathrm{ECRH})$ is used in an ion-temperature-gradient instability (ITG) dominated regime to increase $R / L_{T_{e}}$ in order to approach the trapped-electron-mode instability (TEM) regime. The radial ECRH deposition location determines to a large degree the effect on $R / L_{T_{e}}$. Accompanying scale-selective turbulence measurements at perpendicular wavenumbers between $k_{\perp}=4-18 \mathrm{~cm}^{-1}\left(k_{\perp} \rho_{s}=0.7\right.$ - 4.2) show a pronounced increase of large-scale density fluctuations close to the ECRH radial deposition location at mid-radius, along with a reduction in phase velocity of large-scale density fluctuations. Measurements are compared with results from linear and non-linear flux-matched gyrokinetic (GK) simulations with the gyrokinetic code GENE. Linear GK simulations show a reduction of phase velocity, indicating a pronounced change in the character of the dominant instability. Comparing measurement and non-linear GK simulation, as a central result, agreement is obtained in the shape of radial turbulence level profiles. However, the turbulence intensity is increasing with additional heating in the experiment, while gyrokinetic simulations show a decrease.
\end{abstract}

PACS numbers: 52.70.Gw, 52.25.Os, 52.35.Ra, 52.55.Fa

\section{INTRODUCTION}

The efficiency of a future fusion experiment such as ITER or DEMO will be determined largely by two factors: its susceptibility to magnetohydrodynamic (MHD) instabilities and the transport caused by microturbulence [1]. The design scenario for ITER operation is based on MHD stability and good confinement provided by the $\mathrm{H}$-mode $[2,3]$. The latter is characterized by steep gradients in the plasma pressure in the edge region, often called the pedestal. The resulting pedestal top values for density and temperature set the boundary condition for the bulk profiles, whose properties are determined further inside by the core turbulence. Together, these factors set the confinement and the efficiency of a fusion reactor. Hence, turbulence and its associated transport has not only to be understood from a basic physics point of view, but it is of particular importance to develop good confidence in predictions of turbulent transport. In view of ITER, which will start in L-mode operation where no strong MHD activity is expected, reliable predictions are crucial for an efficient start and fast interpretation of first results.

The main fluctuating quantities in a magnetic confinement fusion plasma are the density $\tilde{n}$, electron and ion temperatures $\tilde{T}_{e, i}$, plasma potential $\tilde{\varphi}$ and the magnetic field $\tilde{B}$. However, strong fluctuation amplitudes do not necessarily cause strong radial transport, since the cross-phase between the above quantities also plays a major role. As yet, a complete characterization is impossible since it would require measurement of fluctuation amplitudes, i.e. power spectra, cross-phases between fluctuating quantities and phase velocities $v_{\mathrm{ph}}$, and all of these for different structure sizes at the same location. It is self-evident that in order to validate gyrokinetic codes, as many of the above quantities as possible must be measured.

*Electronic address: tim.happel@ipp.mpg.de
In general, core turbulence can exist over a large interval of characteristic size scales perpendicular to the magnetic field $\left(k_{\perp} \rho_{s}=0.1-50\right.$ with $\left.\rho_{s}=\sqrt{m_{i} T_{e}} /(e B)\right)$. Taking $\rho_{s}=2 \mathrm{~mm}$, which is typical for magnetic confinement fusion plasmas at mid-radius, structures are about $0.1 \mathrm{~mm}$ to $10 \mathrm{~cm}$ large. Dominant instabilities at these structure sizes are ion temperature gradient modes (ITG) and trapped electron modes (TEM) at large scales $\left(k_{\perp} \rho_{s} \approx 0.1-2\right)$ and electron temperature gradient modes (ETG) at small scales $\left(k_{\perp} \rho_{s} \gg 1\right)$. Further potentially confinement degrading instabilities mainly found in spherical tokamak configurations are microtearing modes (MTM) and parallel velocity gradient (PVG) modes. Experimentally, it is challenging to cover the whole range of structure scales [4-7]. Diagnostics such as Doppler reflectometry, far-infrared scattering, beam emission spectroscopy or phase contrast imaging exist, but these are not necessarily available on all experiments, and do not cover the whole minor plasma radius in devices where they are installed.

Nowadays, to understand turbulent core transport and predict plasma performance, gyrokinetic codes have been and are actively being developed, such as GENE [8], Gs2 [9], GKW [10], GYRo [11], GYSELA [12], GKV/GKV-X [13, 14]. They have been successfully applied to describe core transport properties like profile stiffness [15-20] and (internal) transport barriers [21]. However, while substantial effort has been dedicated to the verification of the codes [22-27], a careful validation including measurements of microturbulence and non-linear simulations, which has to be applied to many different plasma regimes, has only started in the last years [15, 28-34]. The definition of the terms verification and validation are used as in Refs. [30, 35], where verification assesses the degree to which simulations correctly implement a physical model while validation assesses the degree to which a physical model captures "reality" through comparison to experimental measurements.

While good agreement has been found between electric field fluctuations in both frequency and amplitude in global 
simulations [32] and density turbulence level in both directions perpendicular to the magnetic field [29], mixed results have been obtained when comparing the radial dependence of density and temperature fluctuation levels [6, 15, 28]. The scarcity of results, in particular of scale-resolved comparisons $[6,15]$, indicates that these studies are demanding on both experimental and simulation sides, all the more reason to dedicate more effort into validation of gyrokinetic models and their predictions through comparison with microturbulence measurements.

In this paper the properties of density fluctuations and their reaction to changes in the driving terms of microinstabilities are specifically investigated. The information obtained is used to compare with the accompanying gyrokinetic simulations, for which the GENE code [8] is used in its flux-tube version. This comparison of experimental density fluctuation measurements and gyrokinetic simulations is done for the first time in ASDEX Upgrade (AUG). Particular interest is attributed to the reaction of the fluctuations as the turbulence is modified from the ITG towards the TEM regime, which is achieved by applying additional electron cyclotron resonance heating (ECRH) at mid-radius. In this context it has to be noted that although the acronyms ITG and TEM are widely used in literature to distinguish between the two modes, there is no clear boundary where ITG disappears and TEM is excited or vice versa (except for obvious cases like switching off the ITG by using $\nabla T_{i}=0$ in simulations, for example). In contrast, ITG and TEM instabilities can co-exist [17, 36-38], and one can instability can evolve smoothly into the other [36, 38]. Moreover, the ITG instability can be amplified by trapped electrons $[39,40]$. Hence in a real plasma, there is a multitude of effects influencing dominant and subdominant instabilities, and one will not find a black-and-white situation as in theoretical literature. Nevertheless, for the sake of simplicity, the terms ITG and TEM will be used in the present paper. The instabilities will be distinguished based on the sign of their real frequency: the ITG instability propagates into the ion diamagnetic direction while the TEM propagates into the electron diamagnetic direction. In this sense, there is a difference of instability characteristics, which is helpful when discussing experimental and numerical observations.

The experimental technique to investigate the turbulence used in this work is Doppler reflectometry, which has become an increasingly important diagnostic to measure the perpendicular velocity of density fluctuations $u_{\perp}$, radial electric field $E_{r}$ and density turbulence level $\tilde{n}$. The Doppler reflectometry measurement is scale-selective, i.e. the measurement depends on the turbulent structure scale $k_{\perp}$, such that the turbulence response to a change in plasma parameters can be monitored for a variety of different structure sizes, such that measurements of wavenumber spectra become possible [41-46]. In the experiments reported here, the diagnostic scale selectivity has been varied to measure turbulent structures from about 0.3 $\mathrm{mm}$ to $1.5 \mathrm{~cm}$ in a radial range of $\rho_{\text {pol }}=0.5-1.0$, where $\rho_{\text {pol }}$ is the normalized poloidal flux radius, such that about half of the AUG minor radius has been monitored.

This paper is organized as follows: section II introduces the Doppler reflectometry technique and the diagnostic, sections

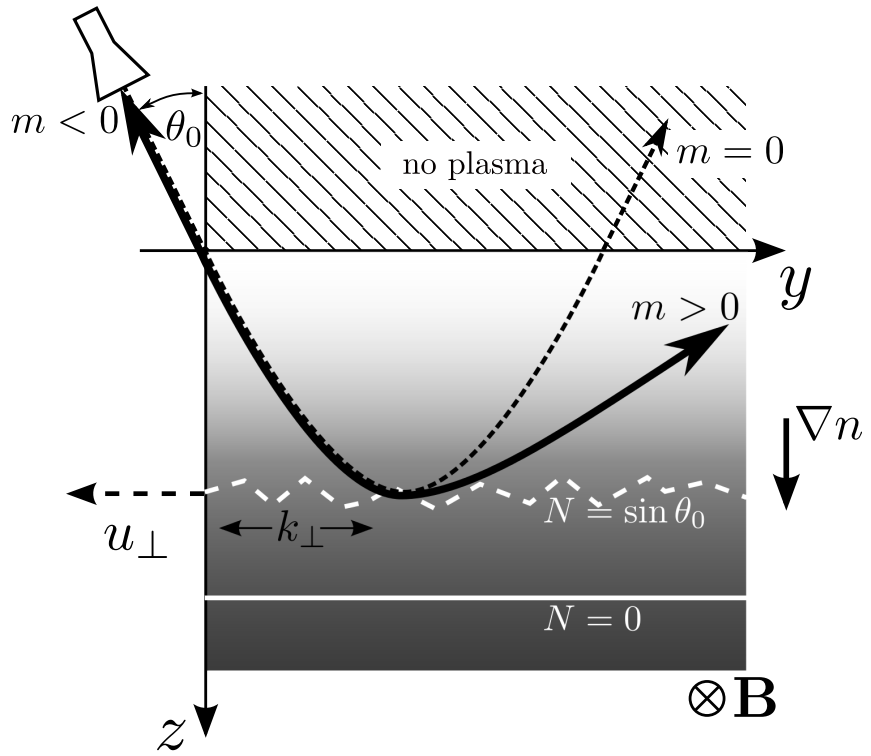

FIG. 1: Schematic drawing of an oblique microwave beam launched into a slab plasma with corrugated cutoff layer (white dashed line), where apart from a direct reflection at order $m=0$ (dashed arrow), scattering occurs at orders $m>0$ (solid line). The grey background indicates the density profile, its gradient points towards positive $z . N$ is the refractive index of the microwave probing beam.

III and IV present the plasma discharge and the turbulence measurements, respectively. The comparison to gyrokinetic numerical simulations is described in section $\mathrm{V}$, followed by a discussion in section VI. Section VII presents a summary and outlook into future work.

\section{MEASUREMENT TECHNIQUE}

Figure 1 shows the principle of Doppler reflectometry [4749] on a plasma in slab geometry. A microwave beam is launched at an angle $\theta_{0}$ with respect to the normal to the isoindex-of-refraction surface. It travels up the density gradient $\nabla n$, which points in positive $z$-direction. Due to the oblique incidence, the beam reflected at the cutoff-layer (or$\operatorname{der} m=0$ ) is not measured. If the cutoff layer is not smooth but corrugated, due to turbulent density fluctuations, the microwave beam is scattered at the cutoff layer (orders $m>0$ ). The antenna is used as a transceiver that selects the Bragg backscattering in the order $m=-1$. The intensity of the wave backscattered under $\theta_{0}$ depends on the spectral Fourier component of the density fluctuations with a wave vector perpendicular to the magnetic field $k_{\perp}$ following Bragg's law

$$
k_{\perp}=2 k_{0} \sin \theta_{0}
$$

where $k_{0}$ is the microwave vacuum wavenumber. In addition, the spectrum of the backscattered signal is Doppler shifted due to the propagation velocity of density fluctuations $u_{\perp}$ and their perpendicular wavenumber $k_{\perp}$ through $f_{\mathrm{D}}=u_{\perp} k_{\perp} / 2 \pi$. 
Hence with the measurement of the Doppler shift $f_{\mathrm{D}}$ and the knowledge of the perpendicular wavenumber $k_{\perp}$, the perpendicular propagation velocity of the turbulent fluctuations can be inferred. The formula for the plasma slab (1) is not valid for plasmas with curved cutoff-layers. In these cases ray or beam tracing has to be used to calculate both the radial position of the backscattering volume $\rho_{\text {pol }}$ and the probed turbulence wavenumber $k_{\perp}$. In this work, the beam tracing code TORBEAM $[50,51]$ was used to calculate the above quantities.

It should be emphasized here that the index " $\perp$ " refers to the direction perpendicular to the magnetic field $\mathbf{B}$ and tangential to the flux surface. Hence, the Doppler reflectometry measurement selects fluctuations with $k_{r}=0$. This definition is different from the one often used in the gyrokinetics community, where the index " $\perp$ " denotes the direction perpendicular to the magnetic field, and not necessarily parallel to the flux surface, with $k_{\perp}^{2} \propto g^{x x} k_{x}^{2}+2 g^{x y} k_{x} k_{y}+g^{y y} k_{y}^{2}$, where $g^{i j}$ are the metric coefficients. For the remainder of the paper, the index " $\perp$ " will be used as stated above, perpendicular to $\mathbf{B}$ and parallel to the flux surface.

The perpendicular velocity $u_{\perp}$ is composed of the plasma background $E \times B$ velocity $v_{E \times B}$ and the phase velocity of turbulent fluctuations $v_{\mathrm{ph}}$ :

$$
u_{\perp}=v_{E \times B}+v_{\mathrm{ph}} .
$$

When the phase velocity of turbulence is small compared to the $E \times B$ velocity, $v_{\mathrm{ph}} \ll v_{E \times B}$, the radial electric field can be deduced from $E_{r}=u_{\perp} B$, where $B$ is the magnetic field strength. Comparison of $E_{r}$ from Doppler reflectometry with $E_{r}$ from heavy ion beam probe measurements at the TJ-II stellarator showed good agreement in the core [52]. Furthermore, comparisons with $E_{r}$ from spectroscopy in W7AS [49] and AUG [53] as well as $E_{r}$ from a ball-pen probe in AUG [54] confirmed that $v_{\mathrm{ph}} \ll v_{E \times B}$ holds, at least in the edge and scrape-off layer regions. In DIII-D, $E_{r}$ was inferred from beam emission spectroscopy, which also measures the turbulence propagation velocity. Comparison with charge exchange recombination spectroscopy measurements also confirm the above statement [55]. However, there are also examples where a non-negligible $v_{\mathrm{ph}}$ has been observed with Doppler reflectometry. One of them is Ohmic plasmas in AUG [56], the other is Tore Supra ion cyclotron resonance heated plasmas [43].

The Doppler reflectometer used in this work is operated in $\mathrm{X}$-mode polarization in a frequency range of $f_{0}=75-105$ $\mathrm{GHz}$ (W-band) [57], giving access to a density range of $2-6$ $\times 10^{19} \mathrm{~m}^{-3}$ for the magnetic field used in this work $(B=2.27$ $\mathrm{T}$ on-axis). A central element in the present studies is the possibility to scan the probed perpendicular wavenumber of the turbulence $k_{\perp}$ via a change in $\theta_{0}$, cf Eq. (1). A schematic of the system in the poloidal cross section of the AUG tokamak is shown in Fig. 2 [58]. Closed and open flux surfaces are shown as solid and dashed lines, respectively. The microwave enters from the right through an oversized $\varnothing 38 \mathrm{~mm}$ waveguide. After emission from one antenna of the bistatic antenna system, the beam is redirected via a plane mirror towards an ellipsoidal mirror. The action of the latter is twofold: first, it controls the tilt angle $\theta_{0}$, and second it serves to focus the beam

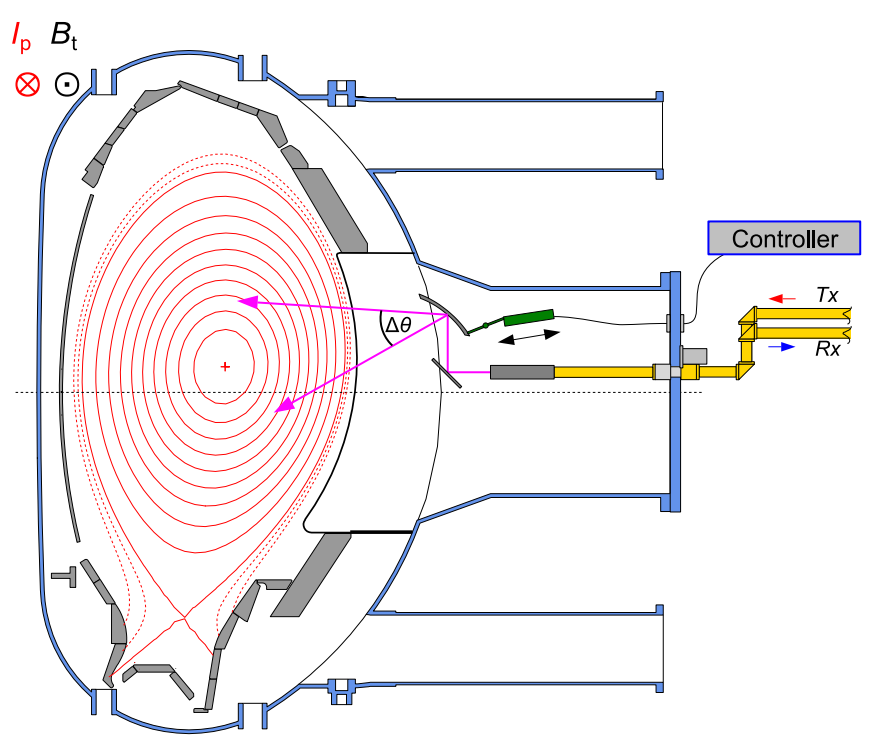

FIG. 2: Poloidal cross-section of the AUG tokamak. The microwave enters through oversized smooth-bore $\varnothing 38 \mathrm{~mm}$ waveguides (TX: transmit) and is emitted by an optimized antenna. After reflection on the lower plane mirror, the beam is steered towards the plasma via the ellipsoidal mirror, where it backscatters off density fluctuations. The $m=-1$ order Bragg-backscattering returns to the ellipsoidal mirror, and is received through RX (receive) by the microwave electronics (not shown). Note the "Controller" for the piezo motor drive, which can steer the ellipsoidal mirror, thus selecting different $k_{\perp}$ of the turbulence via (1).

roughly at $\rho_{\text {pol }}=0.9$ in the plasma, where the wave is partly reflected $(m=0)$ and also scattered $(m \neq 0)$. The Braggbackscattered wave travels back via the ellipsoidal mirror to the lower plane mirror and into the receiver antenna. The latter feeds the beam into the oversized waveguide, which directs it out of the vacuum vessel and into the reflectometer electronics. The rotation of the ellipsoidal mirror is achieved via an in-vessel linear piezoelectric drive, indicated in green along with the double arrow. The linear motion of the piezoelectric stage is converted to a rotation of the ellipsoidal mirror via a linkage incorporating eight ball-bearings. The angular range achieved via rotation of the mirror is $\Delta \theta \approx 35^{\circ}$, corresponding to a perpendicular wavenumber range of roughly $k_{\perp} \approx 0-25$ $\mathrm{cm}^{-1}$. Since the wave is focussed to the cutoff layer with an optimum beam size $[49,59,60]$, the spectral resolution is optimized and typical values of $\Delta k_{\perp} \approx 2.0-2.2 \mathrm{~cm}^{-1}$ are obtained, depending on the probing beam frequency $f_{0}$. For more details on the Doppler reflectometer hardware, the reader is referred to Refs. [57, 61].

\section{PLASMA DISCHARGE DETAILS}

All the discharges reported in this paper were performed in the ASDEX Upgrade (AUG) tokamak and operated in the high-confinement regime (H-mode). Both plasma density and magnetic field strength (on-axis $B=2.27 \mathrm{~T}$ ) were chosen such that the spatial coverage of the Doppler reflectometer 


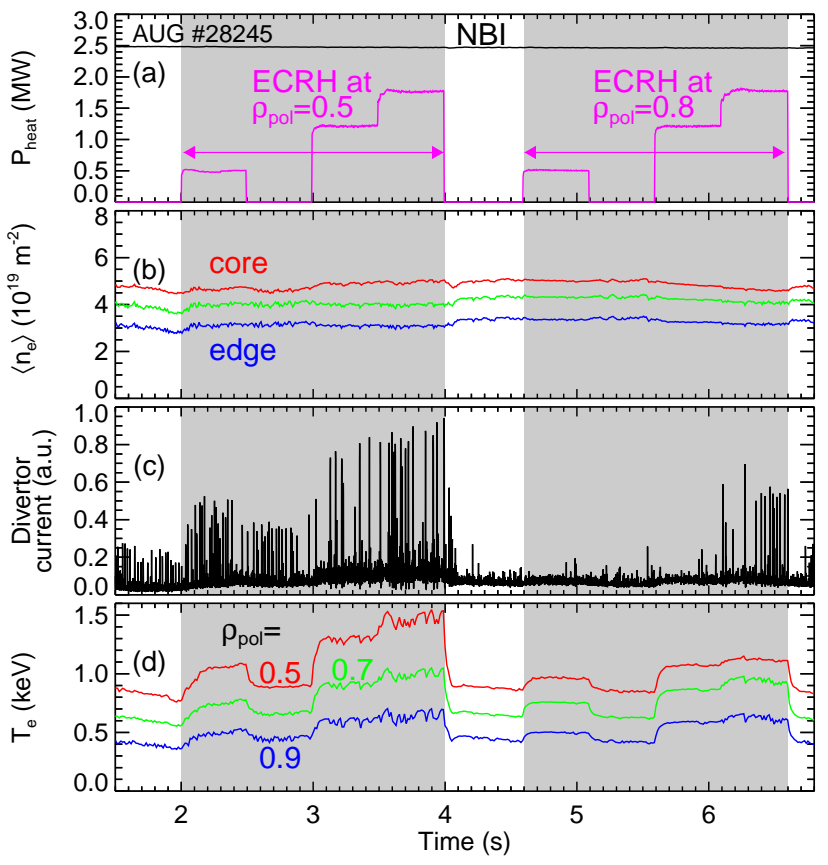

FIG. 3: Time traces of shot \#28245. (a) Heating power $P_{\text {heat }}$, (b) core to edge line-averaged density $\left\langle n_{e}\right\rangle$, (c) divertor currents showing ELMy behavior at high $P_{\mathrm{ECRH}}$, (d) electron temperature $T_{e}$ at $\rho_{\mathrm{pol}}=$ $0.7,0.8$ and 0.9 .

was between $\rho_{\text {pol }}=0.5$ and 1.0. The main objective was to study the turbulence characteristics in both the ITG and the TEM microinstability regime. The transition can be achieved by changing the deposited electron cyclotron heating (ECRH) power to modify the normalized logarithmic electron temperature gradient $R / L_{T_{e}}=-R \nabla T_{e} / T_{e}$ locally $[6,62,63]$, which is a driving term for the TEM instability.

\section{A. Experiment Description}

Figure 3 shows time traces of a representative plasma discharge for the discharge series reported in this paper. Neutral beam injection with heating power of $2.5 \mathrm{MW}$ is used throughout the discharge to obtain a steady state H-mode plasma (a). In order to modify the temperature gradient, ECRH power steps between 0 and $1.8 \mathrm{MW}$ are used. The ECRH power is deposited at $\rho_{\mathrm{pol}}^{\mathrm{ECRH}}=0.5$ from $2.0-4.0 \mathrm{sec}-$ onds, while from $4.6-6.6$ seconds, the deposition location is $\rho_{\mathrm{pol}}^{\mathrm{ECRH}}=0.8$. The two respective time windows are shaded grey.

As can be seen in Fig. 3(b), no pronounced changes are observed in the line-integrated density $\left\langle n_{e}\right\rangle$ when the heating power changes, apart from a slight increase and decrease of the core chord at high ECRH powers at $\rho_{\text {pol }}^{\mathrm{ECRH}}=0.5$ and 0.8 , respectively. This effect is related to density peaking and will be discussed later. In Fig. 3(c), the divertor shunt current, a monitor for ELM activity, shows that the ELM activity increases with heating power. Therefore all analyses in the following are ELM synchronized, meaning that for both
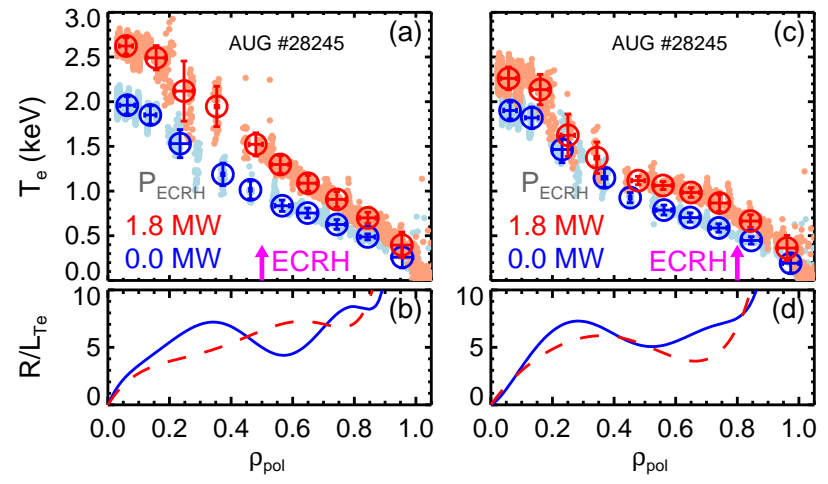

FIG. 4: (a) Electron temperature profiles without (blue) and with (red) additional 1.8 MW ECRH at $\rho_{\mathrm{pol}}^{\mathrm{ECRH}}=0.5$. Note $\nabla T_{e}$ is increased at $\rho_{\text {pol }}>0.5$, resulting in an increase of $R / L_{T_{e}}$ in that region, depicted in (b). (c) and (d) are the same as (a) and (b) for $\rho_{\mathrm{pol}}^{\mathrm{ECRH}}=0.8$. Since $T_{e}$ increases and $\nabla T_{e}$ remains unaffected, $R / L_{T_{e}}$ decreases almost in the whole radial range.

background profile and Doppler reflectometry data analysis, only the inter-ELM phases are taken. The electron temperature $T_{e}$ is depicted in the lower plot, where the influence of the stepped heating power can be clearly observed. Note that for ECRH deposition at $\rho_{\mathrm{pol}}^{\mathrm{ECRH}}=0.5$, the change in $T_{e}$ at $\rho_{\text {pol }}=0.5$ is larger than for $\rho_{\text {pol }}^{\text {ECRH }}=0.8$.

\section{B. Impact of additional ECRH on $T_{e}$ and $R / L_{T_{e}}$}

In Fig. 3(d) it is observed that change in $T_{e}$ is stronger if ECRH is deposited at $\rho_{\text {pol }}^{\mathrm{ECRH}}=0.5$ than 0.8 . The effect of additional ECRH on the electron temperature profiles measured by ECE is shown in Fig. 4. In (a) the radial $T_{e}$ profiles are shown for the cases without ECRH $(t=2.5-3.0 \mathrm{~s}$ in Fig. 3) and with $1.8 \mathrm{MW} \mathrm{ECRH}$ at $\rho_{\mathrm{pol}}^{\mathrm{ECRH}}=0.5(t=3.5-4.0 \mathrm{~s}$ in Fig. 3). As additional ECRH is switched on at mid-radius (red points in (a)), a $T_{e}$ increase across the whole profile is observed. The driving term for TEM turbulence, $R / L_{T_{e}}$, is calculated via a polynomial fit to the $T_{e}$ profile and shown in (b) for the case without ECRH (solid) and with 1.8 MW ECRH (dashed). It can be observed that $R / L_{T_{e}}$ can be increased substantially around the ECRH deposition radius. Further inside, at $\rho_{\text {pol }}<0.4, R / L_{T_{e}}$ decreases. This is due to the fact that inside the ECRH deposition radius, the $T_{e}$ profile rises such that the gradient observed in the profile without ECRH is maintained. This effect becomes more clear for ECRH deposition at $\rho_{\mathrm{pol}}^{\mathrm{ECRH}}=0.8((\mathrm{c}-\mathrm{d})$, cf $t=5.1-5.6 \mathrm{~s}$ and $t=6.1-6.6 \mathrm{~s}$ in Fig. 3), where the whole profile is lifted by ECRH. Consequently, in (d), $R / L_{T_{e}}$ is decreased almost in the whole radial region. Note the similarity of $R / L_{T_{e}}$ for the respective cases without ECRH (solid lines in (b) and (d)), which shows that no history is kept in the plasma discharge.

Figure 5 shows the time window from 2 to 4 seconds, where ECRH steps (a) are applied at $\rho_{\mathrm{pol}}^{\mathrm{ECRH}}=0.5$. The electron temperature at different radii follows $P_{\mathrm{ECRH}}(\mathrm{b})$. In (c), the temporal evolution of the main driving term of TEM and ETG 

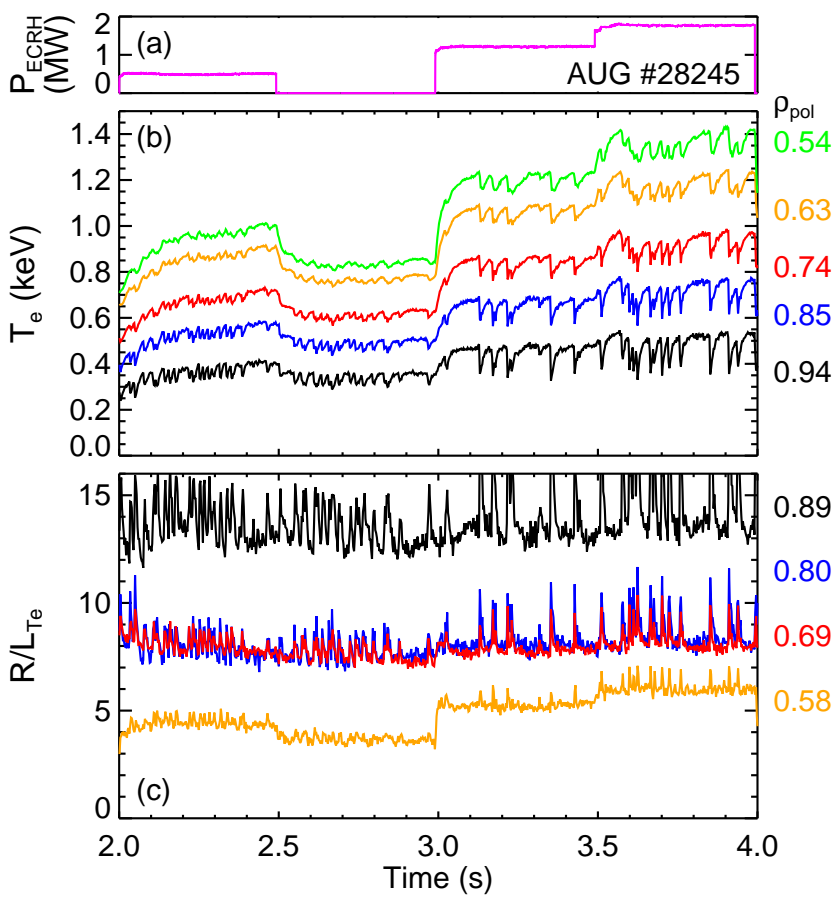

FIG. 5: Time traces of (a) ECRH power, which is deposited at $\rho_{\mathrm{pol}}^{\mathrm{ECRH}}=0.5$ (b) $T_{e}$ and (c) $R / L_{T_{e}}$ at different radial positions. At $\rho_{\text {pol }}=0.58$, the turbulence drive increases with increasing ECRH power, while further outside, it is unaffected. The dips in (b) (spikes in (c)) are due to type I ELMs, which are excluded from the analysis.

turbulence, $R / L_{T_{e}}$, is shown for different radial positions in the plasma. The derivative used in $R / L_{T_{e}}$ has been calculated by the difference between neigboring ECE channels. Although the absolute value obtained with this method is subject to a rather large uncertainty, the trend observed is reliable and serves to give an impression of the effect of ECRH on the turbulence drive term. $R / L_{T_{e}}$ is most affected by $P_{\mathrm{ECRH}}$ at the innermost position $\left(\rho_{\text {pol }}=0.58\right)$, while further outside it remains roughly constant. Hence the most prominent change in direct turbulence measurements - if any - is to be expected at radial positions close to $\rho_{\mathrm{pol}}=0.58$. Note the dips in $T_{e}$ are due to type I ELMs. This temporary decrease of $T_{e}$ during the ELM cycle causes an immediate rise in $R / L_{T_{e}}$ (spikes). As mentioned above, these phases are excluded from the following analysis.

Equivalent time traces, but for ECRH deposition at $\rho_{\mathrm{pol}}^{\mathrm{ECRH}}=$ 0.8, are shown in Fig. 6. Without additional ECRH $(t=5.1$ $-5.6 \mathrm{~s}$ ), the same value $R / L_{T_{e}} \approx 4$ at $\rho_{\text {pol }} \approx 0.6$ is found as in Fig. 5, which shows that no memory from previous heating phases in the discharge is kept. For the $\rho_{\text {pol }}^{\mathrm{ECRH}}=0.8$ case, however, the additional ECRH has a reverse effect on $R / L_{T_{e}}$ : the driving term is reduced as ECRH power is added to the discharge. This is consistent with the observations in Fig. 4, where additional heating at $\rho_{\mathrm{pol}}^{\mathrm{ECRH}}=0.8$ increases $T_{e}$ but not $\nabla T_{e}$ for $\rho_{\text {pol }}<0.8$, reducing $R / L_{T_{e}}$. The onset of the TEM instability is at $R / L_{T_{e}, \text { crit }} \approx 5$ (cf Sec. V A), such that no pronounced changes in turbulence activity are expected with
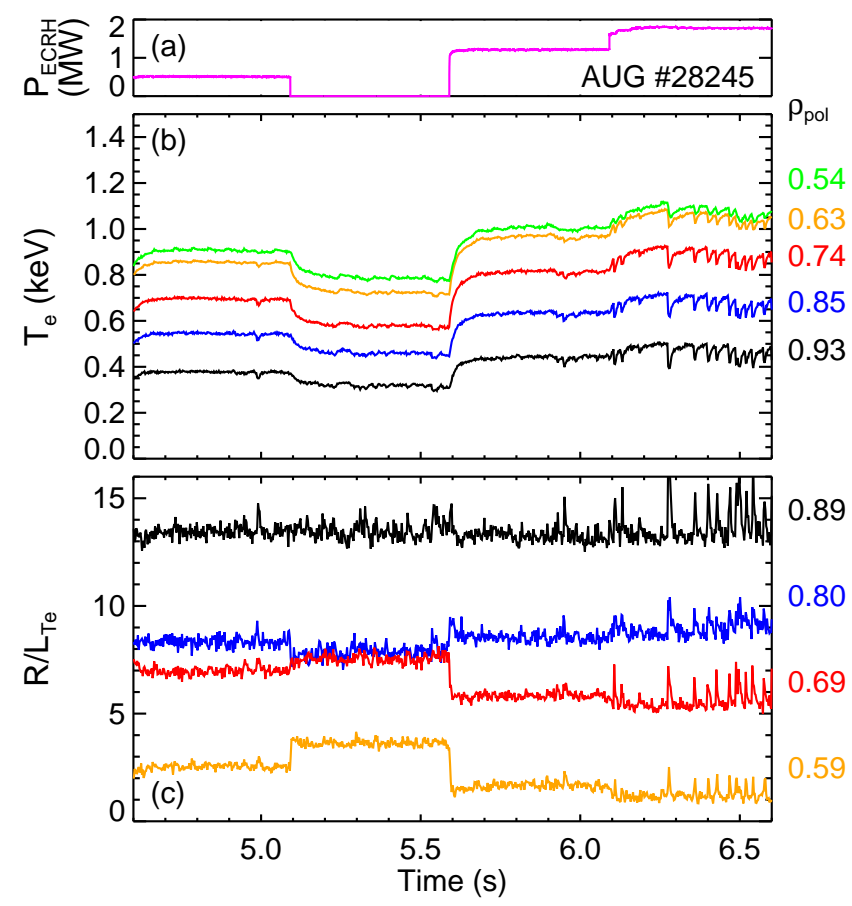

FIG. 6: Same as Fig. 5 for ECRH deposition at $\rho_{\text {pol }}^{\text {ECRH }}=0.8$. In (c), $R / L_{T_{e}}$ is reduced inside of the ECRH power deposition radius $\left(\rho_{\text {pol }}=0.69\right.$ and 0.59$)$

$\rho_{\text {pol }}^{\mathrm{ECRH}}=0.8$, while for $\rho_{\mathrm{pol}}^{\mathrm{ECRH}}=0.5$, an increase in TEM activity might be observed due to a stronger driving term $R / L_{T_{e}}$.

This will be investigated in more detail in Sec. V.

\section{Impact of additional ECRH on plasma profiles}

As described before, additional ECRH has been deposited at $\rho_{\text {pol }}^{\mathrm{ECRH}}=0.5$ and 0.8 at different power levels $(0.5,1.2$ and $1.8 \mathrm{MW})$. For simplicity, in this section only the extreme cases $\left(P_{\mathrm{ECRH}}=0\right.$ and $\left.1.8 \mathrm{MW}\right)$ are considered. Fig. 7(a) shows density profiles before and after switching on of $1.8 \mathrm{MW}$ additional ECRH at $\rho_{\text {pol }}^{\mathrm{ECRH}}=0.5$ corresponding to time windows $2.5-3.0 \mathrm{~s}$ (blue, no ECRH) and $3.5-4.0 \mathrm{~s}$ (red, 1.8 MW ECRH) in Fig. 3. The data shown is from the intervals $2.65-2.95 \mathrm{~s}$ and $3.65-3.95 \mathrm{~s}$ in order to only investigate the plasma once it has reached the new equilibrium. The circles are calculated mean values of the individual measurements at each Thomson scattering (TS) channel [64]. The TS channel at $\rho_{\text {pol }}=0.7$ was not properly calibrated and thus shows a slightly elevated value. Hence, in the following, it is excluded from the profile analysis and from the determination of gyrokinetic code input parameters. For the edge profiles, data from the edge TS and the Lithium beam systems [65] were used.

As ECRH is added to the discharge at $\rho_{\text {pol }}^{\mathrm{ECRH}}=0.5$, a pronounced increase of density at radii inside of the ECRH deposition radius can be observed. Towards the edge, the density profiles overlap. Fig. 7(b) shows the difference between the 

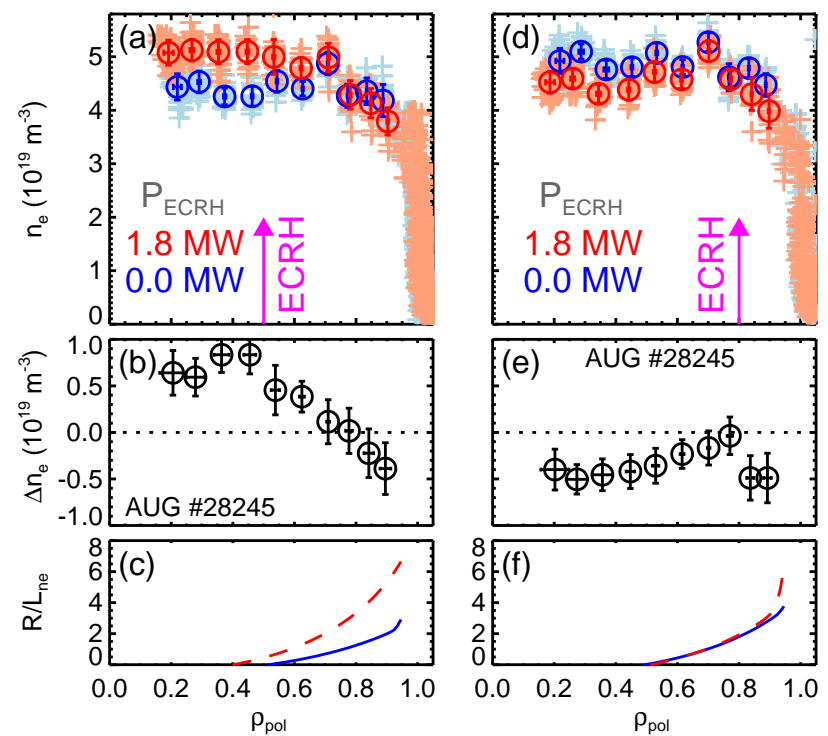

FIG. 7: (a) Density profiles without (blue) and with (red) additional ECRH at $\rho_{\mathrm{pol}}^{\mathrm{ECRH}}=0.5$. The circles are the mean values for each Thomson scattering measurement channel. (b) The difference between density profiles with and without additional ECRH at $\rho_{\mathrm{pol}}^{\mathrm{ECRH}}=0.5$ shows an increase in central density while the edge density remains the same, hence density peaking is observed, as shown in (c). Panels (d), (e) and (f) are the same as (a), (b) and (c), but with ECRH at $\rho_{\text {pol }}^{\mathrm{ECRH}}=0.8$. No change in peaking is observed as ECRH is added (f).

density profiles with and without additional ECRH, $\Delta n_{e}$. The increase of core density, accompanied with a slight edge density reduction, indicates that the discharge is moving from a clear ITG regime towards a situation with increased importance of the TEM instability [66]. The normalized logarithmic gradient $R / L_{n_{e}}$ is depicted in (c), where an increase with ECRH deposition can be observed (dashed line).

Figure 7(d) shows density profiles for the time windows $5.25-5.55 \mathrm{~s}$ and $6.25-6.55 \mathrm{~s}$, which is the analogue to (a), but the ECRH deposition location is now $\rho_{\text {pol }}^{\mathrm{ECRH}}=0.8$. In this case, no density peaking is observed. On the contrary, the profile with ECRH has slightly reduced core density. The difference of both density profiles, $\Delta n_{e}$, is depicted in fig. 7(d). No changes in $R / L_{n_{e}}$ are observed between the two heating regimes (f).

It is important to note that for $\rho_{\mathrm{pol}}^{\mathrm{ECRH}}=0.5$, the density profile is more peaked than without ECRH (cf Fig. 7(b)), meaning that $R / L_{n}$ increases with additional ECRH. This can have an influence on the prevailing microinstability: $R / L_{n}$ is a stabilizing term for temperature gradient driven modes (ITG, ETG) $[67,68]$, provided it is comparable to $R / L_{T}$, which is not the case here. However, $R / L_{n}$ is also a destabilizing term for TEMs [21, 69-71]. Hence, with increased heating power and the changes reported to both $R / L_{T_{e}}$ (cf Sec. III B) and $R / L_{n}$, a more pronounced influence of TEMs can be expected for $\rho_{\mathrm{pol}}^{\mathrm{ECRH}}=0.5$, but not for $\rho_{\mathrm{pol}}^{\mathrm{ECRH}}=0.8$.

Figure 8 shows radial profiles of the toroidal plasma rotation $v_{\text {tor }}$ measured with charge exchange recombination spec-
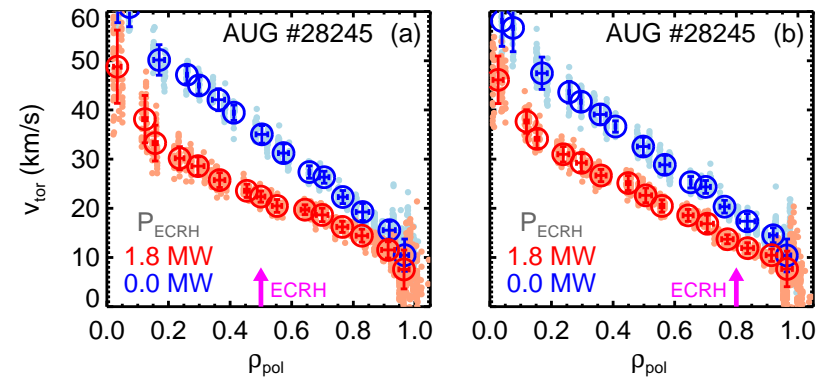

FIG. 8: Toroidal rotation profiles without (blue) and with (red) 1.8 MW ECRH. For both heating locations $\left(\rho_{\text {pol }}^{\text {ECRH }}=0.5(a)\right.$ and $\left.0.8(b)\right)$, $v_{\text {tor }}$ decreases when ECRH is added. The effect is stronger for the inner ECRH deposition location (a).
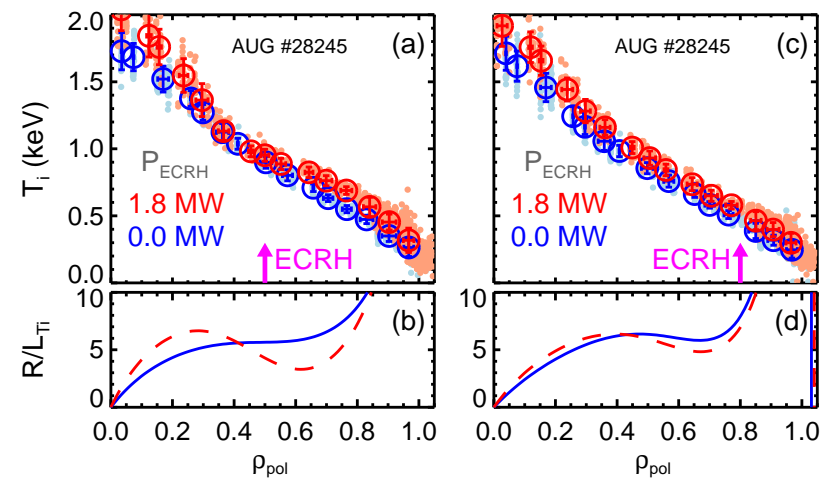

FIG. 9: (a) Ion temperature profiles without (blue) and with (red) additional $1.8 \mathrm{MW} \mathrm{ECRH} \mathrm{at} \rho_{\mathrm{pol}}^{\mathrm{ECRH}}=0.5$. Note $\nabla T_{i}$ is reduced and $T_{i}$ is increased at $\rho_{\text {pol }}>0.5$, resulting in a decrease of $R / L_{T_{i}}$, depicted in (b). (c) and (d) is the same as (a) and (b) for $\rho_{\text {pol }}^{\mathrm{ECRH}}=0.8$, where an effect of the additional ECRH is hardly visible.

troscopy (CXRS) on boron ions [72]. In all cases $v_{\text {tor }}$ is peaked and decreases monotonously towards the plasma edge. For ECRH at locations $\rho_{\text {pol }}^{\mathrm{ECRH}}=0.5$ (a) and $0.8(\mathrm{~b})$, a decrease of $v_{\text {tor }}$ is observed. This decrease is more pronounced in (a), indicating that higher central $T_{e}$ leads to a stronger reduction in $v_{\text {tor }}$. This is consistent with studies of the influence of ECRH on toroidal rotation in $\mathrm{H}$-mode discharges in AUG [73].

The effect of additional ECRH on the ion temperature profiles measured by CXRS on boron ions is shown in Fig. 9. The profiles without additional ECRH $(t=2.5-3.0 \mathrm{~s}$ and $t=5.1-5.6 \mathrm{~s}$ ) are similar. As additional ECRH is switched on at mid-radius (red points in (a)), an increase of $T_{i}$ at $\rho_{\text {pol }}^{\mathrm{ECRH}}<\rho_{\text {pol }}<0.9$ is observed. At the same time, its gradient decreases around and outside of the heating deposition radius. Hence $R / L_{T_{i}}$ is reduced, which is depicted in (b). It is interesting to note that the flat $T_{i}$ in this region must be caused by an increased transport. This is counterintuitive to the fact that ITG turbulence is driven by $R / L_{T_{i}}=-R \nabla T_{i} / T_{i}$. However, the critical ion temperature gradient $R /\left.L_{T_{i}}\right|_{\text {crit }} \propto 1+T_{i} / T_{e}$ [67], such that ITG turbulence is more unstable as ECRH is added, which reduces the second term on the rhs. This could explain the increase in $\chi_{i}$ and the related flattening of $T_{i}$. For the case of added heating at $\rho_{\text {pol }}^{\mathrm{ECRH}}=0.8(\mathrm{c})$, a slight $T_{i}$ increase over 
the whole plasma radius is visible without any strong changes to $\nabla T_{i}$. Hence, $R / L_{T_{i}}$ is barely affected (d).

Finally, in the radial range of interest for this work $\left(\rho_{\text {pol }}=\right.$ $0.5-0.8)$, the ratio of electron to ion temperature is $T_{e} / T_{i} \approx$ 1.0 without additional ECRH, while it increases up to $T_{e} / T_{i} \approx$ 1.4 with $P_{\mathrm{ECRH}}=1.8 \mathrm{MW}$. In the same radial region the electron-ion collision frequency $v_{\mathrm{ei}}$ is found between $v_{\mathrm{ei}} /\left(c_{s} / R\right)=1.0$ and 2.5 at $\rho_{\mathrm{pol}}=0.5$ and 0.8 for $P_{\mathrm{ECRH}}=0$, where $c_{s}$ and $R$ are the ion sound velocity and major radius, respectively. For $P_{\mathrm{ECRH}}=1.8 \mathrm{MW}, v_{\mathrm{ei}} /\left(c_{s} / R\right)$ is between 0.5 and 1.5 , hence it is reduced by approximately a factor of two when 1.8 MW ECRH is added. The impurity density profile has been measured for boron and carbon impurities and is roughly constant over the plasma radius with values around $1.5 \times 10^{17} \mathrm{~m}^{-3}$, giving a value for $Z_{\mathrm{eff}}=1.2$ for the above radii, which is used in the section on simulations (Sec. V).

\section{TURBULENCE MEASUREMENTS}

In order to monitor scale-resolved turbulence changes with different ECRH powers, the ellipsoidal mirror of the Doppler reflectometer was moved on a shot-to-shot basis, changing the probing beam tilt angle and thus the measured $k_{\perp}$ (cf Sec. II). Moreover, in each shot, scans in probing beam frequency between $f_{0}=74.7$ and $103.5 \mathrm{GHz}$ were performed within $100 \mathrm{~ms}$ time windows, such that profile measurements from $\rho_{\text {pol }}=0.5$ to 1.0 are available for each probing beam tilt angle [74]. Altogether, this gives experimental radial profiles of $u_{\perp}$ and $\tilde{n}$ for the different electron temperature logarithmic gradients as obtained by changing the ECRH positions and heating powers.

\section{A. Perpendicular propagation velocity of the turbulence}

As introduced in Sec. II, through measurement of the Doppler shift $\omega_{\mathrm{D}} \approx u_{\perp} k_{\perp}$, the perpendicular velocity of density fluctuations $u_{\perp}\left(k_{\perp}\right)=v_{E \times B}+v_{\mathrm{ph}}\left(k_{\perp}\right)$, where $v_{E \times B}$ is the $E \times B$-velocity and $v_{\mathrm{ph}}$ is the phase velocity of the turbulence, can be measured. It is important to note that the dependence of $u_{\perp}$ on $k_{\perp}$ originates exclusively from $v_{\mathrm{ph}}$, and thus is a direct consequence of the turbulence propagation. For the plasma discharges presented in this paper, $u_{\perp}\left(k_{\perp}\right)$ has been obtained for all the ECRH power steps at both heating locations, resulting in a total of eight profiles. For each profile, the measurements are available at different structure sizes ranging from $k_{\perp}=4-18 \mathrm{~cm}^{-1}$.

Figure 10 shows $u_{\perp}$ for different additional ECRH heating powers at $\rho_{\mathrm{pol}}^{\mathrm{ECRH}}=0.5$ (indicated by the magenta arrow, without ECRH (a), 0.5 MW (b), 1.2 MW (c) and 1.8 MW (d)) and different turbulence scales (color-coded). All profiles range from approximately $\rho_{\text {pol }}=0.5$ to the separatrix and show positive $u_{\perp}$ from mid-radius to the pedestal top and a well in the edge gradient region with a minimum around $\rho_{\text {pol }}=0.99$. Assuming $u_{\perp}$ is dictated to a large degree by $E_{r}$, the profile shape is consistent with previous observations in AUG Hmodes [53] and also other tokamaks [75]. The perpendicular flow in the edge region AUG has been shown to be in agreement with neoclassical theory [76]. The positive values in the core result from the toroidal NBI momentum input while the edge well is caused by the ion pressure gradient [77]. The $u_{\perp}$-minimum has also been observed further inside in smaller experiments $[42,52,78]$, and it has been proposed that the absolute distance to the separatrix (roughly $3 \mathrm{~cm}$ ) is the main player rather than a distance in normalized magnetic flux [52].

In Fig. 10(a) (no additional ECRH), large, intermediate and small structure sizes (blue, green and red) propagate with the same $u_{\perp}$, such that $v_{\mathrm{ph}} \ll v_{E \times B}$ can be assumed. Another explanation could be an independence of $v_{\mathrm{ph}}$ on $k_{\perp}$. This is the case for a linear dispersion relation $\omega\left(k_{\perp}\right)$, which cannot be excluded here. Adding 0.5 MW ECRH power barely changes the $u_{\perp}$-profile, only the $u_{\perp}$ well becomes marginally deeper. With the addition of 1.2 MW ECRH power (c), the situation changes: while small and intermediate scales continue to propagate with roughly $5 \mathrm{~km} / \mathrm{s}$ around mid-radius, large structures experience a deviation and propagate slower, an effect which is increased for $P_{\mathrm{ECRH}}=1.8 \mathrm{MW}$ (d). This change in $u_{\perp}$ at $\rho_{\text {pol }}<0.8$ with $P_{\mathrm{ECRH}}>1 \mathrm{MW}$ could be related to a change in phase velocity $v_{\mathrm{ph}}$ of large-scale turbulent structures, which would point into the electron-diamagnetic direction, consistent with the usual propagation direction of TEMs. The change in $u_{\perp}$ can be compared to the expected phase velocity of the turbulence, which is generally of the order of $v_{\mathrm{ph}} \approx 3 \rho_{s} c_{s} / R[56,79]$. At $\rho_{\mathrm{pol}}=0.6$ this gives a value of $v_{\mathrm{ph}} \approx 1.6 \mathrm{~km} / \mathrm{s}$. This is less than, but of the same order of magnitude, as the experimentally observed difference of 3 $\mathrm{km} / \mathrm{s}$, showing that the observed effect could indeed be due to a modification of $v_{\mathrm{ph}}$ of large structures.

Corresponding $u_{\perp}$ profiles for $\rho_{\mathrm{pol}}^{\mathrm{ECRH}}=0.8$ are shown in Fig. 11. As in Fig. 10, $u_{\perp}$ is roughly $5 \mathrm{~km} / \mathrm{s}$ at mid-radius and decreases to values of roughly $-7 \mathrm{~km} / \mathrm{s}$ at the $u_{\perp}$ well, which is again located at roughly $\rho_{\text {pol }}=0.99$. In contrast to the $\rho_{\text {pol }}^{\mathrm{ECRH}}=0.5$ case, no effect in the $u_{\perp}$ profile can be seen when adding ECRH power at $\rho_{\mathrm{pol}}^{\mathrm{ECRH}}=0.8$ on any scale, the profiles remain comparable even when adding 1.8 MW ECRH. Note that no small scales $\left(k_{\perp}>14 \mathrm{~cm}^{-1}\right)$ have been measured at the highest power (d), which is due to a loss of signal from the Doppler reflectometer. This is caused by the combination of two different effects: first, with additional ECRH at $\rho_{\text {pol }}^{\text {ECRH }}=0.8$, the density profile becomes very flat, making reflectometry measurements in general more challenging. Second, the turbulence level for small scales is rather low, such that the combination of flat density profile and low turbulence level causes the backscattered signal to drop below the diagnostic limit.

\section{B. Radial profiles of density turbulence level}

Figure 12 shows turbulence levels $\tilde{n}$ for the four different ECRH heating levels (color-coded) for $\rho_{\text {pol }}^{\mathrm{ECRH}}=0.5$ (cf Fig. 5). Although no absolute turbulence levels are measured by Doppler reflectometry, relative changes are meaningful. In (a), $\tilde{n}$ is shown for the largest investigated turbulence struc- 

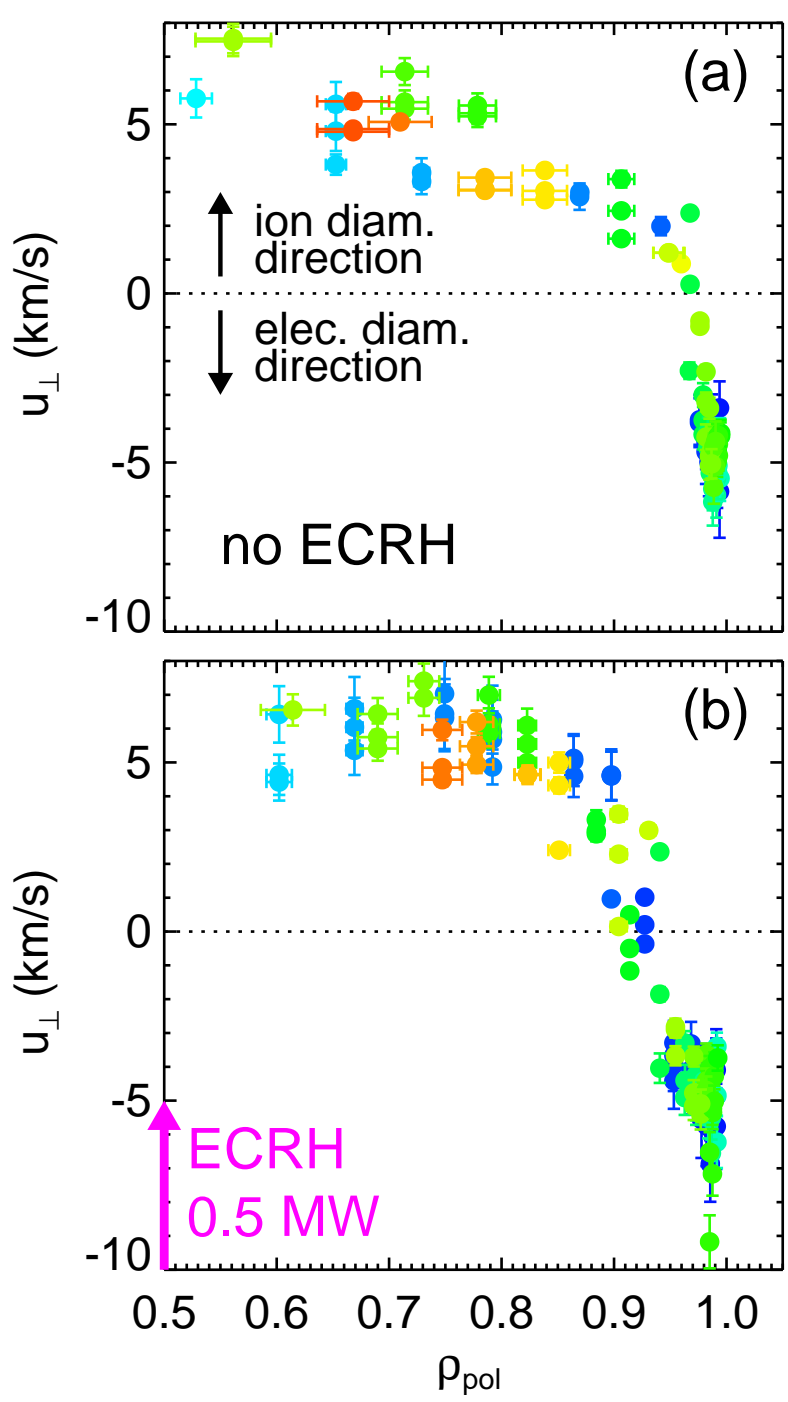

AUG \#28244-7
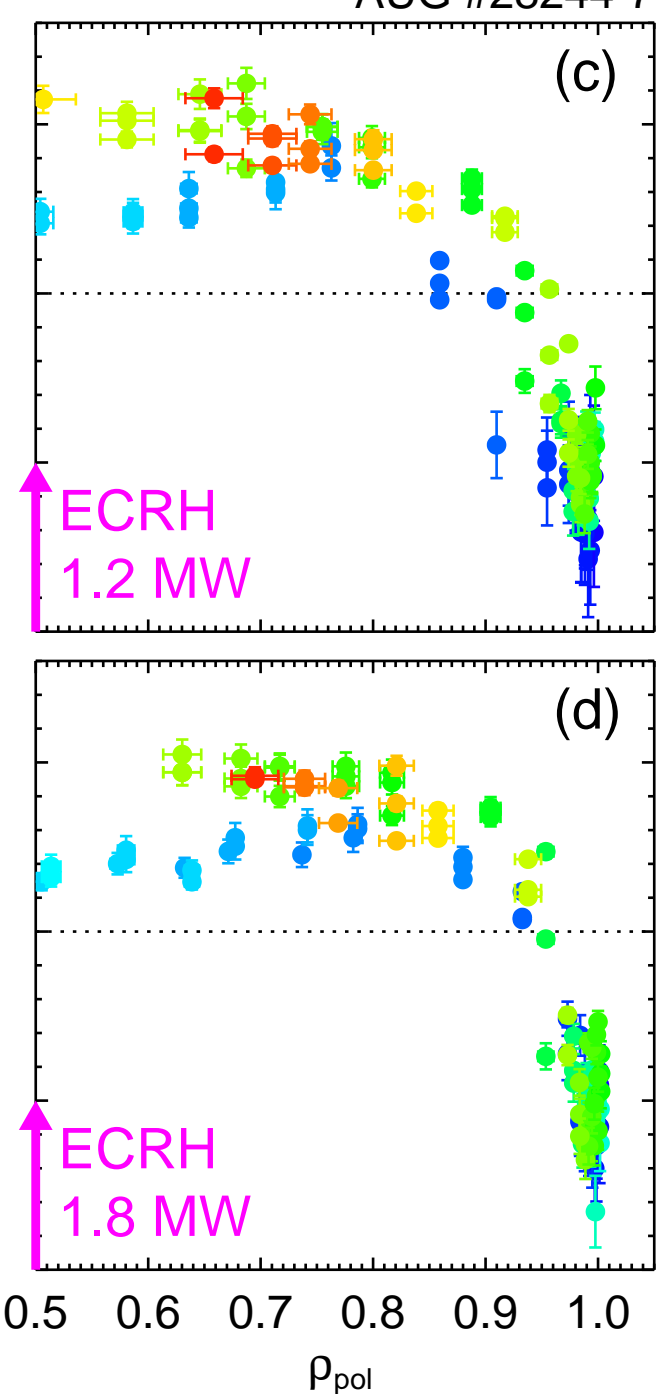

FIG. 10: Perpendicular velocity of density fluctuations for different heating powers. Additional ECRH is deposited at $\rho_{\text {pol }}^{\mathrm{ECRH}}=0.5$.

tures with perpendicular wavenumbers of $k_{\perp}=4-8 \mathrm{~cm}^{-1}$. As a general observation, from the core towards the edge, $\tilde{n}$ increases up to roughly $\rho_{\text {pol }}=0.98$, which has been observed in many tokamaks and stellarators $[80,81]$. In the very edge $\left(\rho_{\text {pol }}=0.99\right)$ a significant reduction of turbulence is observed close to the position of the $E_{r}$ shear layer, consistent with observations in H-mode on many devices [42, 81-84]. An influence of the additional ECRH power on the turbulence level is observed around mid-radius, close to the ECRH deposition location. For ECRH powers less than $1 \mathrm{MW}$ (circles), no difference in the turbulence level profiles can be detected. However, at $P_{\mathrm{ECRH}}>1 \mathrm{MW}\left(R / L_{T_{e}}>5\right)$, an increase in turbulence level in a radial region from $\rho_{\text {pol }} \approx 0.50-0.75$ is detected. Towards the edge, the influence of additional ECRH decreases and the turbulence level profiles are indistinguishable at $\rho_{\text {pol }}>0.75$. The increase in $\tilde{n}$ close to the ECRH deposition radius could be related to the increase in $R / L_{T_{e}}$ as observed in Fig. 5(c), where the largest $R / L_{T_{e}}$ increase is at the innermost observed radius $\left(\rho_{\text {pol }}=0.58\right)$ at $P_{\mathrm{ECRH}}>1 \mathrm{MW}$.

As noted before, smaller turbulence scales were also investigated in the discharge series. The turbulence level profiles for $k_{\perp}=8-13 \mathrm{~cm}^{-1}$ and $k_{\perp}=13-18 \mathrm{~cm}^{-1}$ are shown in Fig. 12(b) and (c), respectively. Similarly, the general shape shows an increase of $\tilde{n}$ from the core towards the edge. At comparable radii, $\tilde{n}$ decreases from large turbulence scales towards smaller scales ((a) to (c)). For the smallest scales investigated, close to the $E_{r}$ shear layer measurements were not possible due to a loss of the Doppler shifted component.

For neither (b) nor (c) an influence of the additional ECRH power on the turbulence level profile can be detected. The growth rates of ITG and TEM instabilities tend to peak around $k_{\perp} \rho_{s}=0.4-0.7$ (cf Sec. V A), which corresponds in these experiments to $k_{\perp}=2-4 \mathrm{~cm}^{-1}$, dropping to larger $k_{\perp}$. Since the turbulence level changes observed at $k_{\perp}=4-8 \mathrm{~cm}^{-1}$ 

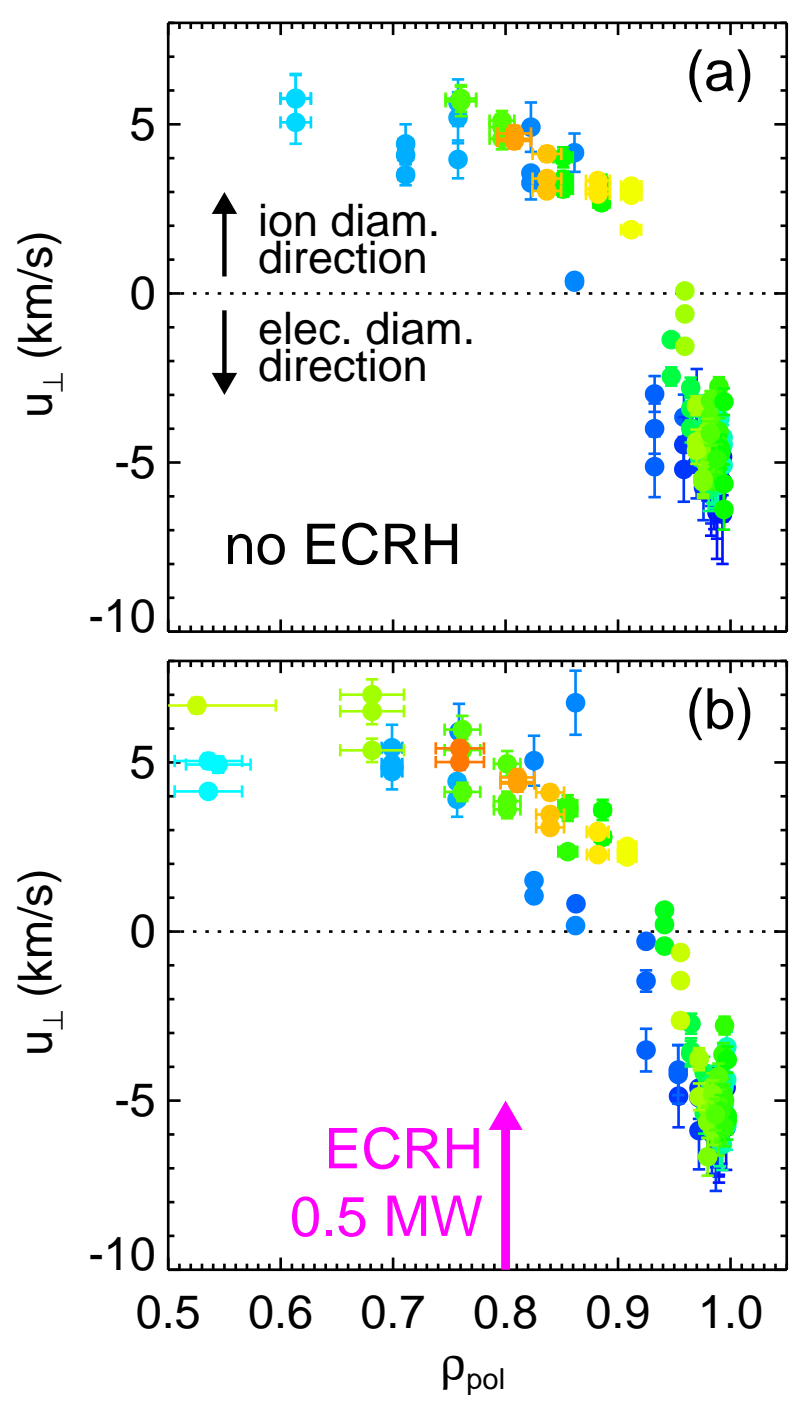

AUG \#28244-7
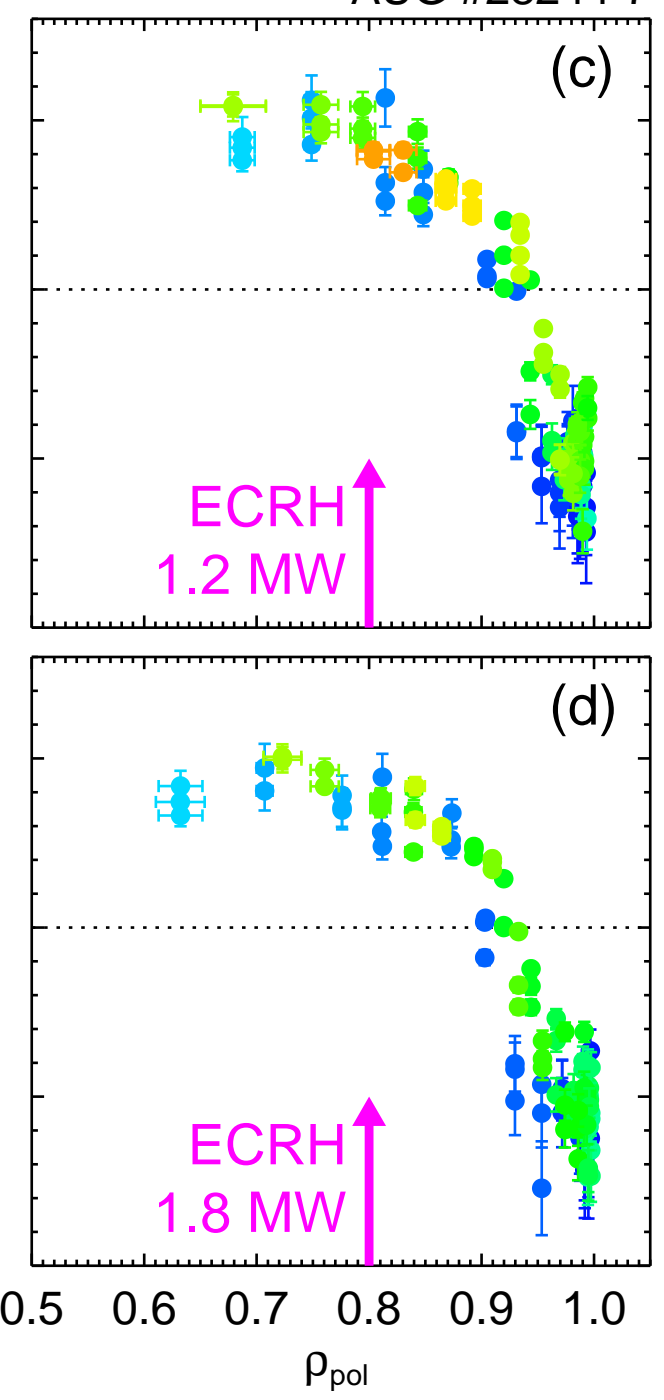

FIG. 11: Perpendicular velocity of density fluctuations for different heating powers. Additional ECRH is deposited at $\rho_{\mathrm{pol}}^{\mathrm{ECRH}}=0.8$.

are strong, but weaker for smaller scales $\left(k_{\perp}=8-13 \mathrm{~cm}^{-1}\right.$ and $k_{\perp}=13-18 \mathrm{~cm}^{-1}$ ), this could point to a change in the properties of the energy cascade with collisionality and turbulence drive, as has been observed both theoretically and numerically [85-87]. To make a clear statement on this point, detailed measurements of wavenumber spectra are necessary, which are not available for the discharges discussed here.

Figure 13 shows density turbulence level profiles $\tilde{n}$ for $\rho_{\text {pol }}^{\mathrm{ECRH}}=0.8$, corresponding to $t=4.6-6.6 \mathrm{~s}$ in Fig. 3 and Fig. 6. As a general observation, for all structure sizes the radial and spectral dependence of turbulence level is the same as in Fig. 12, i.e. an increase from core towards edge with a reduction close to the $E_{r}$ shear layer and a decrease of $\tilde{n}$ from large towards small scales. In contrast, an influence of additional ECRH at $\rho_{\mathrm{pol}}^{\mathrm{ECRH}}=0.8$ on the turbulence level is not observed. This is consistent with Fig. 6(c), where only at $\rho_{\text {pol }}=0.8$ an almost negligible increase in $R / L_{T_{e}}$ is seen, whereas the general trend is a reduction of $R / L_{T_{e}}$. Since in this case $R / L_{T_{i}}$ is the dominant turbulence driving term (ITG dominant), the source of free energy for the turbulence is barely affected for $\rho_{\mathrm{pol}}^{\mathrm{ECRH}}=0.8$, and correspondingly no change in $\tilde{n}$ in Fig. 13 is observed.

To summarize, an effect of additional ECRH on the turbulence quantities $u_{\perp}$ and $\tilde{n}$ is only detected for ECRH with sufficient power $(P>1 \mathrm{MW})$ deposited at mid-radius for largescale turbulence $\left(k_{\perp}=4-8 \mathrm{~cm}^{-1}\right)$. The effect is a more negative phase velocity of turbulent fluctuations $v_{\text {ph }}$ along with an increase in the fluctuation amplitude. 

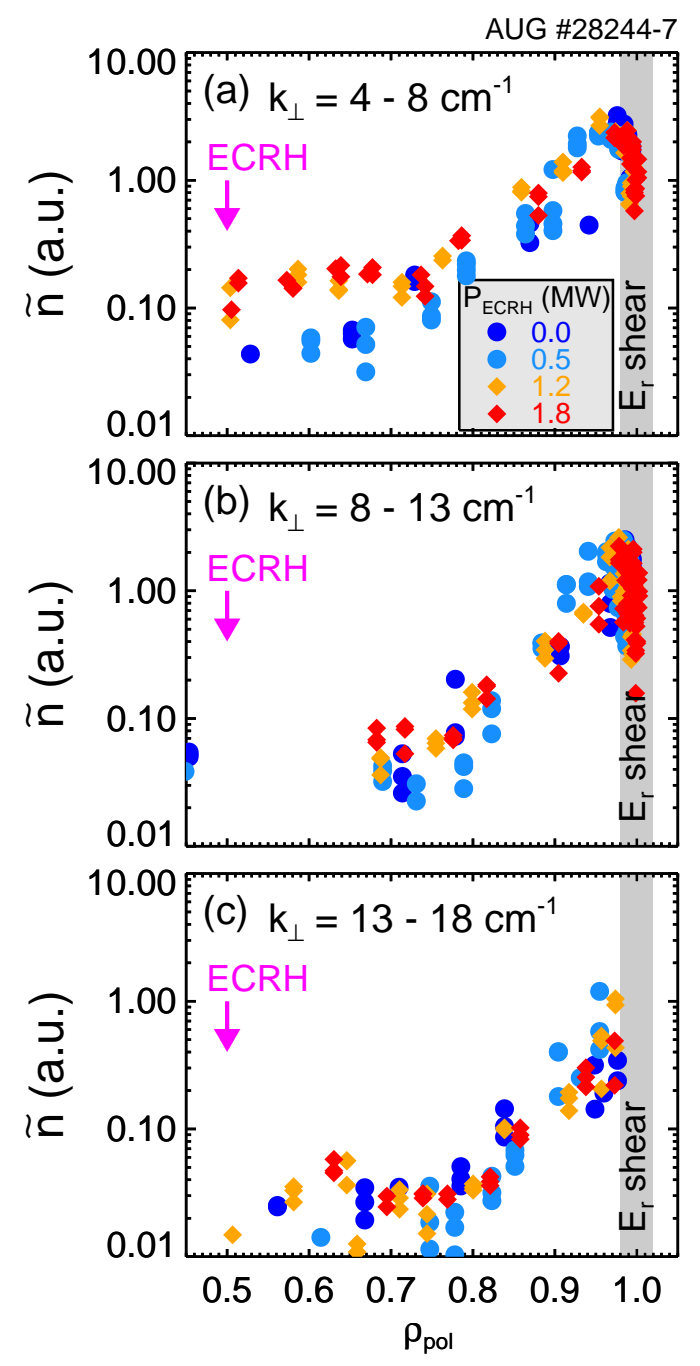

FIG. 12: Radial turbulence level profiles for different structure scales. Large structures $\left((\mathrm{a}), k_{\perp}=4-8 \mathrm{~cm}^{-1}\right)$ show an increase in core turbulence level with increasing ECRH heating power at $\rho_{\mathrm{pCl}}^{\mathrm{ECRH}}=0.5$ (indicated by magenta arrow), while smaller structures ((b), (c)) are barely affected by ECRH. Close to the $E_{r}$ shear layer, the turbulence level drops. At small turbulence scales (c), it even drops below the diagnostic limit.

\section{ASTRA AND GENE MODELING FOR ECRH DEPOSITION AT $\rho_{\text {pol }}^{\mathrm{ECRH}}=0.5$}

Since the strongest effect on all quantities investigated $\left(R / L_{n}, R / L_{T_{i}}, R / L_{T_{e}}, u_{\perp}\right.$ and $\tilde{n}$ [cf Figs. 7, 9, 5, 10 and 12] $)$ was observed for $\rho_{\text {pol }}^{\mathrm{ECRH}}=0.5$ at $1.8 \mathrm{MW}$ additional ECRH power, this case and the one without ECRH will be used for comparison with linear and non-linear simulations using the gyrokinetic plasma turbulence code GENE [8]. To identify the underlying microinstabilities linear simulations were performed. The experimental ion and electron heat fluxes $\left(Q_{i, e}\right)$ and diffusivities $\left(\chi_{i, e}\right)$ were obtained through power balance analysis with the ASTRA code [88], to be subsequently compared to results from non-linear GENE simulations. Apart

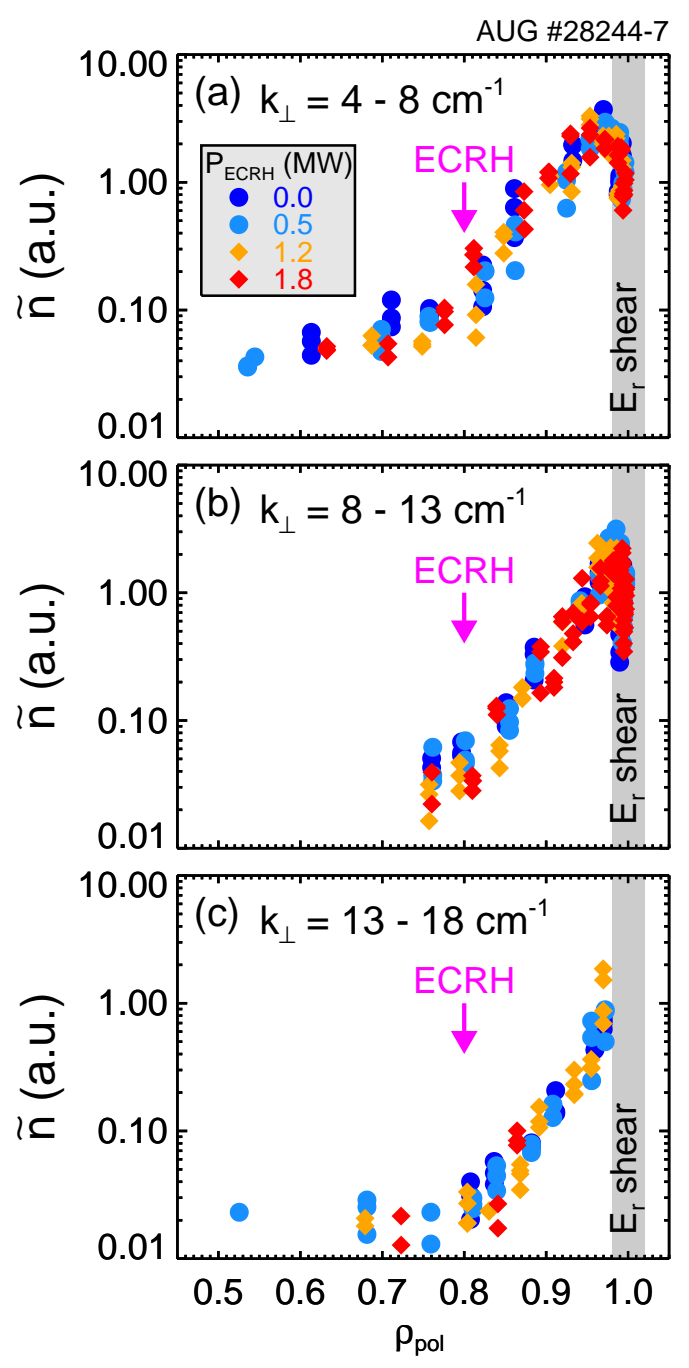

FIG. 13: Same as Fig. 12 for ECRH deposition at $\rho_{\text {pol }}^{\text {ECRH }}=0.8$ (indicated by magenta arrow). Note that no effect of additional electron heating is visible on the density turbulence level profile.

from heat fluxes which are flux-matched to the power balance analysis results, GENE non-linear simulations yield the turbulence level $\tilde{n}$, which will be compared to the experimental results from Sec. IV B. For all linear simulations presented, the resolution is $\{31 \times 32 \times 48 \times 16\}$ in $\left\{x, z, v_{\|}, \mu\right\}$ coordinates, where $\mu$ is the magnetic moment (non-linear: $\{256 \times 128 \times$ $32 \times 48 \times 16\}$ in $\left.\left\{x, y, z, v_{\|}, \mu\right\}\right)$. The non-linear simulation box sizes are $\left\{125 \rho_{s}, 125 \rho_{s},[-\pi, \pi],[-3,3] v_{\mathrm{th}, \alpha},[0,9] T_{\alpha} / B\right\}$ in $\left\{x, y, z, v_{\|}, \mu\right\}$. Here, $\alpha$ denotes the species (electrons or ions). Convergence tests for several cases with double resolution in the perpendicular direction have been done and show no difference to the results presented. Similarly, perpendicular box sizes including several turbulence correlation lengths are used and have been checked for convergence. The results presented are time averaged values after the simulations are converged and have subsequently been run for several autocorrelation times of the turbulence. The experimental equilibrium is processed through the Tracer-EFIT interface [89]. The effective 

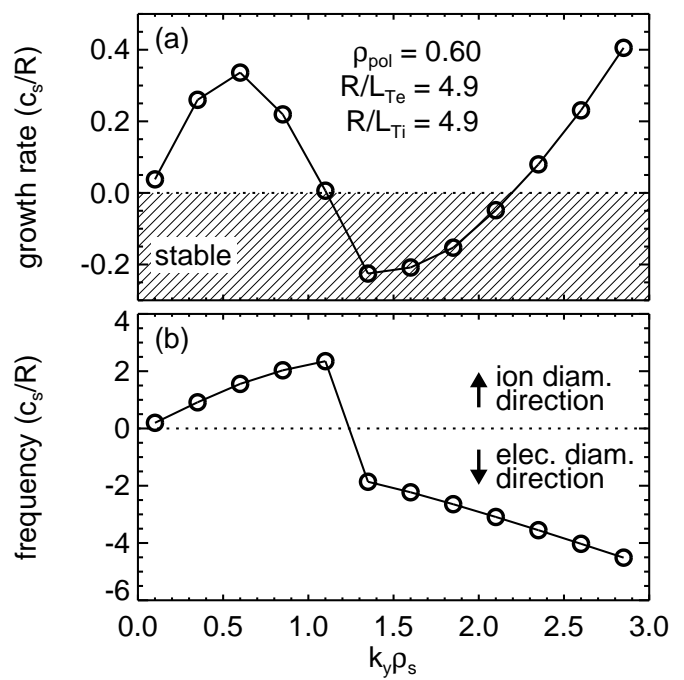

FIG. 14: Results from linear GENE simulations for $R / L_{T_{e}}=R / L_{T_{i}}=$ 4.9. (a) Growth rate showing two unstable branches at large and small structure sizes with a stable region around $k_{\perp} \rho_{s}=1.6$. (b) Frequency of the fastest growing mode. Positive and negative frequencies correspond to drifts into ion- and electron-diamagnetic direction, respectively.

charge is $Z_{\mathrm{eff}} \approx 1.2$. Two kinetic species (ions and electrons) are used with realistic mass ratio $\sqrt{m_{i} / m_{e}} \approx 60$, and electromagnetic effects are included by solving for the parallel component of Ampère's law. Since it has been observed that including impurities as a third kinetic species can impact growth rates even at low $Z_{\text {eff }}$ [31], test cases with three kinetic species were run but yielded only small differences to the two-species results (less than $10 \%$ deviation in growth rates and heat fluxes). Furthermore, it was ascertained in linear and non-linear test cases that the dilution effect from the fast ion density can be neglected. The $E \times B$ shear is approximated from the toroidal velocity of boron impurities (cf Fig. 8) via $\gamma_{\mathrm{E}}=\rho_{t} / q\left(\partial \omega_{\text {tor }} / \partial \rho_{t}\right)$, with $\rho_{t}$ the normalized toroidal flux and $\omega_{\text {tor }}$ the toroidal rotation frequency. This method neglects contributions from the boron poloidal velocity and from the boron pressure gradient (with atomic number $Z=5$ ) to calculate $E_{r}$. For all cases considered, $\gamma_{\mathrm{E}} / \gamma_{\max } \lesssim 0.25$, with $\gamma_{\max }$ the growth rate maximum. Although $\gamma_{\mathrm{E}}$ has been found to be negligible - even an increase of $40 \%$ showed no significant impact on neither growth rates nor heat fluxes - it is included in all simulations presented. For a more extensive description of the simulations and a detailed analysis of parameter scans for the presented discharges, the reader is referred to Ref. [87].

\section{A. Linear gyrokinetic simulations}

In order to identify the fastest growing microinstability (ITG or TEM), linear gyrokinetic simulations have been conducted for normalized structure sizes $k_{y} \rho_{s}=0.1-2.85$ and logarithmic temperature gradients $R / L_{T_{i}}$ and $R / L_{T_{e}}$ spanning the range $1.2-9.8$ each. Figure 14 shows a representative
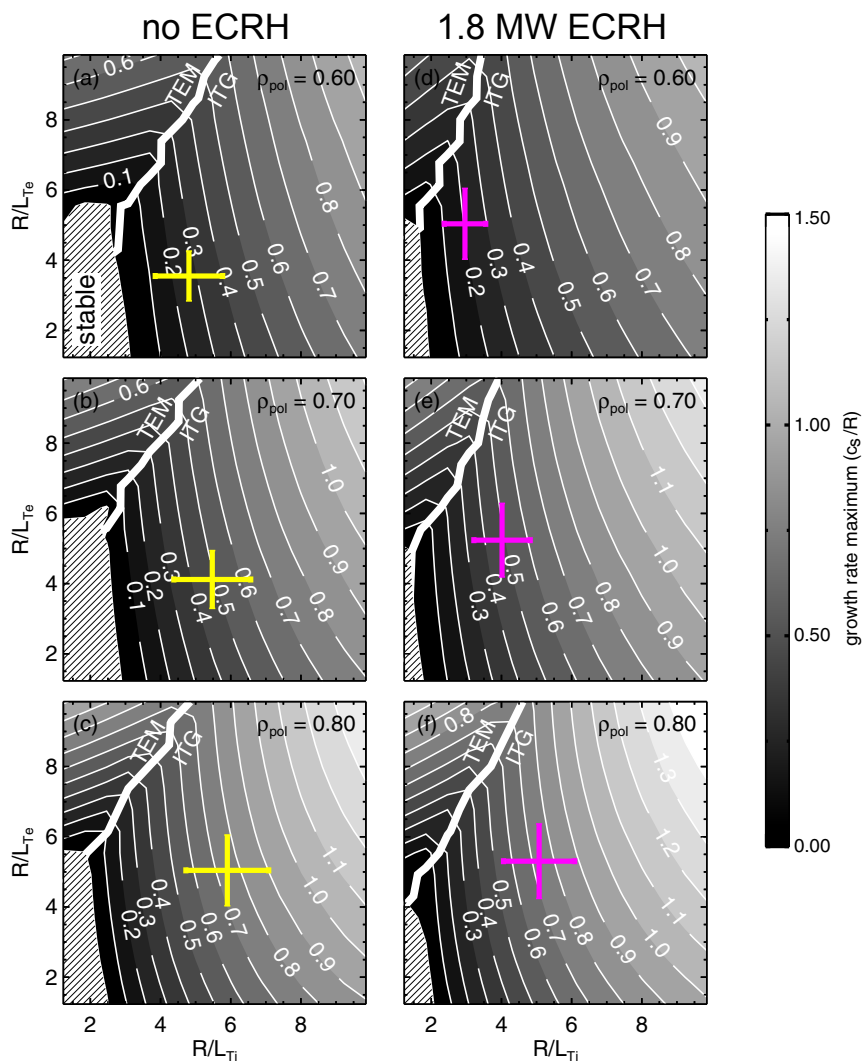

FIG. 15: Maximum growth rate (color coded) for scales $k_{y} \rho_{s}=0.2-1.0$ and its dependence on normalized ion- and electron temperature gradients, $R / L_{T_{i}}$ and $R / L_{T_{e}}$, respectively, for the cases without (a-c) and with (d-f) ECRH. The thick white line denotes the boundary between TEM (upper left) and ITG (lower right) sections, identified via a change of sign of frequency. The yellow and magenta crosses indicate the experimental gradients for the case without and with ECRH, respectively. At the innermost radii $\rho_{\text {pol }}=0.60$ and 0.70 , adding ECRH brings the discharge close to the TEM regime, while further outside $\rho_{\text {pol }}=0.80$, it does not.

result of one linear simulation for $\rho_{\mathrm{pol}}=0.60$ and $R / L_{T_{e}}=$ $R / L_{T_{i}}=4$.9. The growth rate of the fastest growing mode is shown in Fig. 14(a). It is positive for large scales $\left(k_{y} \rho_{s}<1\right)$ and small scales $\left(k_{y} \rho_{s}>2\right)$, and there is a gap for intermediate scales where no unstable mode is found. To identify the dominant microinstability, the frequency of the fastest growing mode has to be considered, as depicted in Fig. 14(b). In GENE, positive and negative frequencies correspond to the ionand electron-diamagnetic drift directions, respectively. For $k_{y} \rho_{s}<1$ the propagation in the ion-diamagnetic direction identifies the ITG mode. For $k_{y} \rho_{s}>2$, propagation is in the electron-diamagnetic direction. Considering the wavenumber range, this instability can be identified as an electron temperature gradient driven mode (ETG).

Figure 15 depicts the growth rate maximum $\gamma_{\max }$ for ITG or TEM instabilities color-coded for different radii $\left(\rho_{\text {pol }}=0.60,0.70\right.$ and 0.80$)$ for the case without ECRH (ac) and with $1.8 \mathrm{MW}$ ECRH (d-f). Background parameters $\left(R / L_{n}, T_{e} / T_{i}, q, \hat{s}, \ldots\right)$ are taken from the respective experi- 
mental situation (without and with ECRH), $\gamma_{\max }$ is calculated in the range $k_{y} \rho_{s}=0.2-1.0$. The largest structures at $k_{y} \rho_{s}=0.1$ have been excluded because they were found to contain contributions from microtearing modes (MTMs). Since, in the presence of ITGs, MTMs may not contribute substantially to heat transport [90], and the corresponding large perpendicular scales are not measured by the Doppler reflectometer, the choice to exclude $k_{y} \rho_{s}=0.1$ does not influence the conclusions. In the line-filled region in the lower left corners, both ITGs and TEMs are stable, i.e. their growth rates are negative. For largest $R / L_{T_{i}}$ and $R / L_{T_{e}}$ the highest growth rates are observed (upper right corner). The thick white line indicates where the frequency of the fastest growing mode changes sign, it thus describes the boundary between ITG (lower right) and TEM (upper left) instabilities. A general observation is that ITG is the preferred instability, in that for $R / L_{T_{e}}=R / L_{T_{i}}$, the ITG is the fastest growing mode. This is also observed in studies of impurity transport and intrinsic rotation on Alcator C-Mod [33, 91]. The crosses localize the gradients in the experiment. Their sizes indicate the error bars in the respective quantities, which are assumed to be $20 \%$ of the measurement. The growth rate maximum increases with radius for both without and with ECRH.

Comparing the cases without and with ECRH, it becomes apparent that the boundary between TEM and ITG turbulence becomes steeper with ECRH, at least for $\rho_{\text {pol }}=0.60$ and 0.70 . This means that with ECRH, the ITG mode is more dominant in comparison to the case without ECRH. This is a particularly interesting effect which can be explained by the fact that both $T_{e} / T_{i}$ and $R / L_{n}$ increase substantially with electron heating, such that the ITG mode becomes more unstable, which is also reflected in the smaller stable region in the lower left parts of (d) and (e). Still, since with ECRH the logarithmic gradients $R / L_{T_{e}}$ and $R / L_{T_{i}}$ are changed (yellow and magenta crosses), the experimental situation is now closer to a regime with possible influence of the TEM instability, at least for $\rho_{\text {pol }}=0.60$ and $0.70((\mathrm{~d})$ and $(\mathrm{e}))$. It is important to note that while a distinction between ITG and TEM is certainly adequate for practical reasons, in reality they can co-exist, even with a smooth transition from one to the other [38]. Furthermore, trapped electrons are not exclusively related to the TEM instability. Instead, they have been shown to play a major role in the ITG instability $[39,40]$. For more details on the simulation results, the reader is referred to Ref. [87].

Figure 16 shows $v_{\text {ph }}$ color-coded without (a) and with 1.8 MW ECRH (b) for $\rho_{\mathrm{pol}}=0.60$, corresponding to the simulations from Fig. 15(a) and (d). Again, the experimental point in $R / L_{T_{e}}$ - and $R / L_{T_{i}}$-parameter space is marked by the yellow and magenta crosses. In general, $v_{\mathrm{ph}}$ is of the order of several $100 \mathrm{~m} / \mathrm{s}$ and negative and positive for the TEM and ITG instability, respectively. In the case without ECRH (a) and for the experiment parameters, $v_{\mathrm{ph}} \approx 580 \mathrm{~m} / \mathrm{s}$, while for the case with ECRH (b), $v_{\mathrm{ph}} \approx 340 \mathrm{~m} / \mathrm{s}$. Hence from linear gyrokinetic simulations a reduction of $v_{\mathrm{ph}}$ is expected, which is qualitatively consistent with the experimental observation (cf Sec. IV A, Fig. 10(d)). However, quantitatively, the observed change of roughly $3 \mathrm{~km} / \mathrm{s}$ from the experiment is not recovered here. Furthermore, the simple estimate of $v_{\mathrm{ph}} \approx 3 \rho_{s} c_{s} / R \approx 1.6$

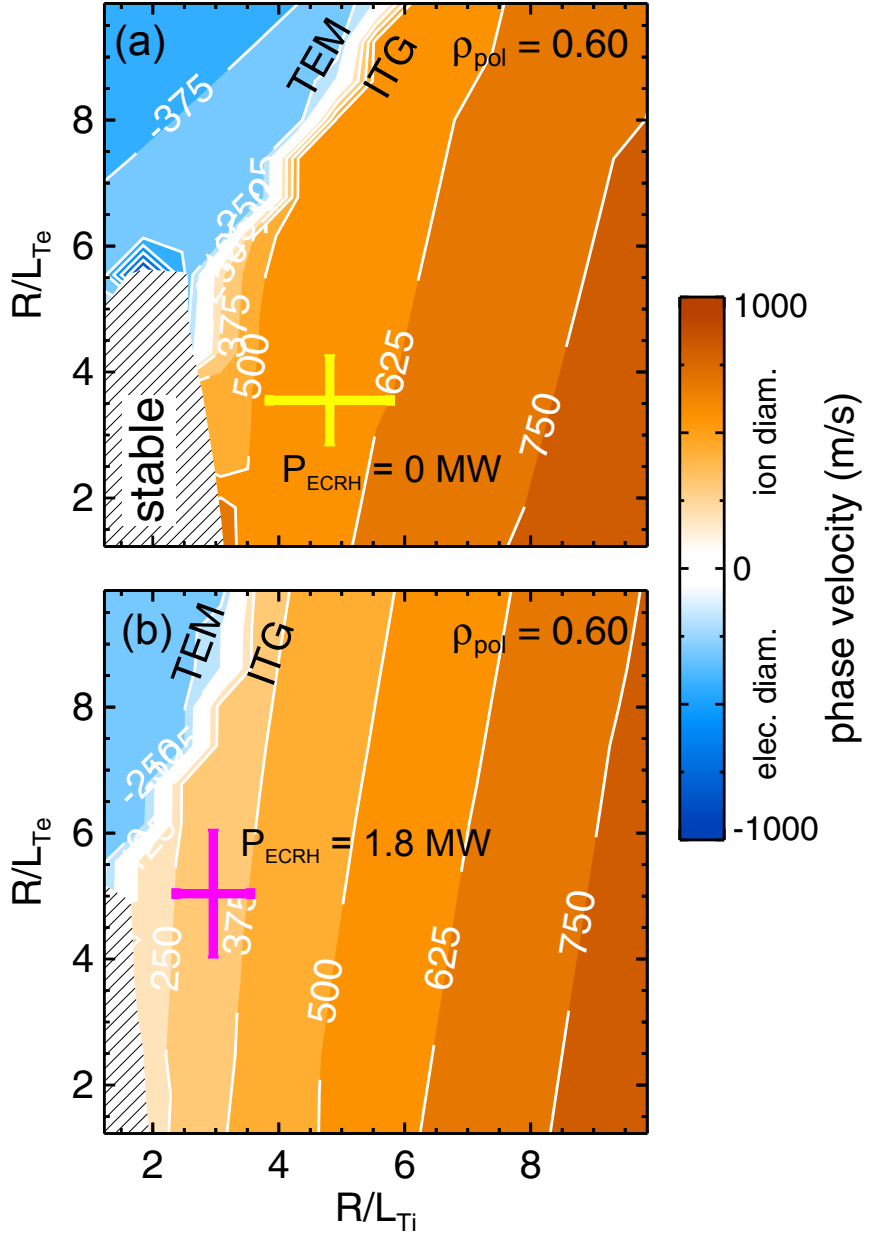

FIG. 16: Phase velocities $v_{\text {ph }}$ obtained via linear gyrokinetic simulation at $\rho_{\mathrm{pol}}=0.60$. The yellow and magenta crosses indicate the experimental gradients for the case without and with ECRH, respectively. In both cases without (a) and with 1.8 MW ECRH (b), $v_{\mathrm{ph}}$ is of the order of several $100 \mathrm{~m} / \mathrm{s}$. A reduction of $v_{\mathrm{ph}}$ with additional ECRH is observed.

$\mathrm{km} / \mathrm{s}[56,79]$ seems to overpredict $v_{\mathrm{ph}}$ compared to the gyrokinetic simulations.

Concluding, it can be observed that adding 1.8 MW ECRH to the discharge has the effect of approaching the boundary between TEM and ITG turbulence. However, through the changes in parameters other than ion- and electron temperature gradients, the parameter space changes such that it becomes difficult to reach a situation in which the TEM is the dominant instability. From the present linear gyrokinetic simulations, it can be inferred that a clear transition into a dominant TEM regime can only be achieved if $R / L_{T_{e}}$ and $R / L_{T_{i}}$ are changed without strongly modifying $T_{e}$ and $T_{i}$. Experimentally, this is a challenging task since an increase in $\nabla T_{e}$ requires a change to $T_{e}$, which affects the ITG stability through $T_{e} / T_{i}$ and thus destabilizes the ITG mode. 


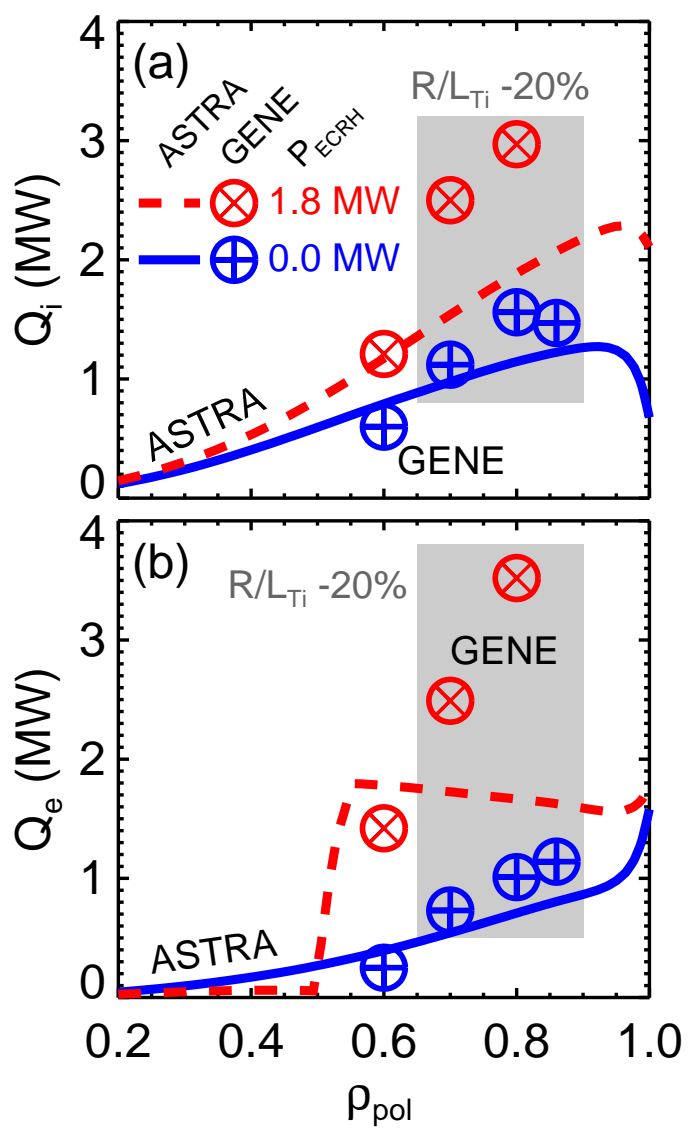

FIG. 17: ASTRA power balance analysis (lines) and non-linear gyrokinetic simulation results (plus symbols and crosses). (a) Ion heat flux, (b) electron heat flux. The gyrokinetic simulation results at $\rho \geq 0.7$ (indicated by the gray shaded box) are from runs in which $R / L_{T_{i}}$ is decreased by $20 \%$ in order to match the heat fluxes from power balance analysis. For details refer to the text.

\section{B. Non-linear gyrokinetic simulations}

In order to evaluate and interpret non-linear gyrokinetic simulations, a two-step approach has been taken: first, the experimental heat fluxes are calculated via power balance analysis with the ASTRA code [88]. Then GENE non-linear simulations are conducted, modifying the ion temperature gradient $R / L_{T_{i}}$ within error bars such that a good match to the experimental heat fluxes is obtained. Only $R / L_{T_{i}}$ has been modified since in all cases investigated with linear simulations, ITG is predicted as the fastest growing mode (cf Sec. V A). Due to the fact that ITG drives both ion and electron heat fluxes, both channels are reasonably well matched to the experiment. In the second step, the density fluctuation level $\tilde{n}$ from the gyrokinetic simulations is compared with the measurements from Doppler reflectometry. A more detailed analysis with emphasis on the theoretical part of the non-linear gyrokinetic simulations including parameter scans and the analysis of spectral properties and cross-phases between turbulent quantities is out of the scope of this paper and will be presented elsewhere [87].

For the different heating powers used in the experiment,
Fig. 17 shows the ion- and electron heat fluxes as obtained from power balance analysis (lines). Note that with $P_{\mathrm{ECRH}}=$ $1.8 \mathrm{MW}$ deposited at $\rho_{\text {pol }}^{\mathrm{ECRH}}=0.5, Q_{e}(\mathrm{~b})$ increases substantially at that radial position. Apart from the ASTRA calculations, results from non-linear gyrokinetic simulations in the fluxtube limit are shown as plus symbols and crosses for zero and 1.8 MW additional input power, respectively. Simulations were performed for scales $k_{\perp} \rho_{s}<3$. For the present comparison, the restriction to larger scales than the characteristic scales of the electron temperature gradient (ETG) mode is justified because in the experiment only the large-scale turbulence is affected by the changes to the background gradients.

In order to match the heat fluxes, $R / L_{T_{i}}$ had to be reduced by $20 \%$ at radii $\rho_{\text {pol }} \geq 0.7$ for both the $P_{\mathrm{ECRH}}=0$ and 1.8 MW cases. These results are indicated in Fig. 17 by the gray shaded box shown in both frames. Since ITG modes cause the main heat transport, no changes in $R / L_{T_{e}}$ have been applied. However, for the (not shown) outer radius at $P_{\mathrm{ECRH}}=1.8$ MW, linear results show that small-scale turbulence (ETG) could be important. It has been observed recently that if small scales are important for heat transport, they can have an effect on heat fluxes derived from large scales [92], which would add a source of uncertainty to the simulations. Furthermore, for this radial position even large-scale simulations take an order of magnitude longer to converge than all the other points. Therefore, and due to limited resources, it was decided to focus on the other seven radial points. In general, good agreement is obtained between GENE heat fluxes and the power balance result from ASTRA for the $P_{\mathrm{ECRH}}=0$ case, while the $P_{\mathrm{ECRH}}=1.8 \mathrm{MW}$ case is somewhat overpredicted. Taking into account the stiffness of the profiles, the overprediction is not very pronounced, since a further minor change to the gradients would match the experimental heat fluxes. The phase velocities obtained from the turbulence fields of the non-linear simulations have been compared to the linear phase velocities. In all cases, propagation in the ion-diamagnetic direction is observed as in linear simulations, and also the reduction of $v_{\mathrm{ph}}$ for $P_{\mathrm{ECRH}}=1.8 \mathrm{MW}$ with respect to the $P_{\mathrm{ECRH}}=0$ case is recovered (cf Fig. 16). However, the absolute $v_{\mathrm{ph}}$ values from non-linear simulations are consistently below the linear results. This point is not understood and may be related to insufficient statistics for this particular post-processing purpose. A final answer in this context is hence left for future work.

To visualize the above changes in $R / L_{T_{i}}$ necessary to obtain realistic heat fluxes, Fig. 18 shows the $T_{i}$ profile (against the normalized toroidal flux radius $\rho_{\mathrm{tor}}$ ) for the $P_{\mathrm{ECRH}}=0$ case. Light blue are the measurements from CXRS [72] (10 ms time resolution), while the black circles give the mean of each channel in the whole time window and error bars are the standard deviation of the respective measurements. The fit to the experimental data is shown in blue, the final GENE input gradients to obtain the heat fluxes from Fig. $17, P_{\mathrm{ECRH}}=0$, in red. The inset shows a zoom to the radial position $\rho_{\text {tor }}=0.56$ (equivalent to $\rho_{\text {pol }}=0.70$ ). Locally, a change of $-20 \%$ in $R / L_{T_{i}}$ is within the degrees of freedom available to the profile fitting routine. Note that although a change to $R / L_{T_{i}}$ can be obtained by changing both $T_{i}$ and $\nabla T_{i}$, in this work only a change to $\nabla T_{i}$ is considered, since the measurement of the 


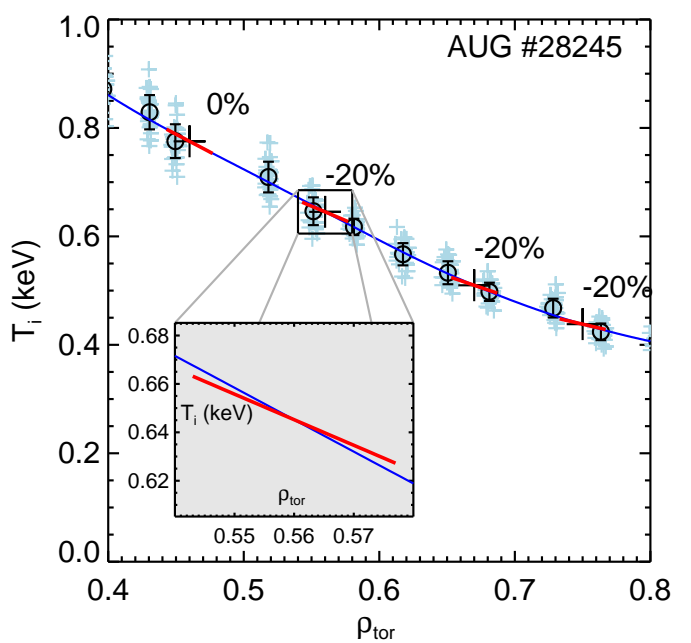

FIG. 18: Radial $T_{i}$ profile. GENE input gradients required to achieve experimental heat flux values are shown in red. The inset shows a zoom to one of the simulated radii, where the difference in gradient can be observed.

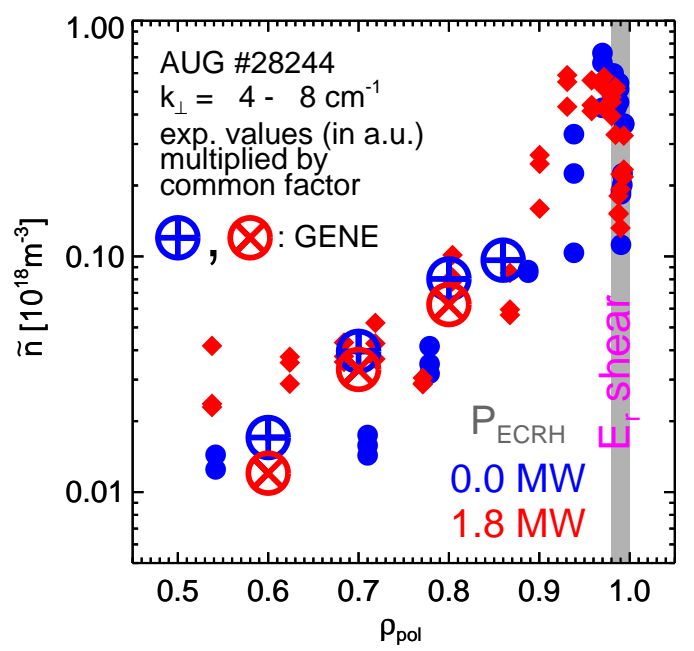

FIG. 19: Comparison of GENE and experimental turbulence levels. Experimental turbulence levels have been scaled by a common factor to match GENE absolute turbulence levels.

absolute value of $T_{i}$ is assumed to be more reliable than its gradient.

It should be emphasized that although a change of $-20 \%$ to $R / L_{T_{i}}$ at a specific location in the $T_{i}$ profile required to match experimental heat fluxes might be inside the experimental uncertainties, it is certainly not if it has to be applied continuously to a large fraction of the profile.

Figure 19 compares the radial turbulence level profile for the largest scales investigated $\left(k_{\perp}=4-8 \mathrm{~cm}^{-1}\right)$ with results from the corresponding gyrokinetic simulations (plus symbols and crosses). Note that a good $k_{\perp}$-selectivity of the diagnostic is given by the fact that the spectral resolution is $\Delta k_{\perp} \approx 2 \mathrm{~cm}^{-1}$ (cf Sec. II and Ref. [61]). The GENE results have been analyzed on the low-field side in the outer midplane at the height of the magnetic axis, where the Doppler reflectometer measurements are taken (cf lower arrow in Fig. 2). The poloidal variation of measurements (different $k_{\perp}$ are measured at different launch angles) is sufficiently small for $k_{\perp}=4-8$, such that no poloidal dependence in the analysis of the turbulence fields is considered. However, the gyrokinetic results have been filtered in $k_{\perp}$-space to match the scales which have been measured by the Doppler reflectometer diagnostic. The experimental points, which are measured in arbitrary units, have been multiplied by a common factor to give a reasonable match to the absolute $\tilde{n}$ values obtained with the GENE simulation. At first sight, the basic trend measured with Doppler reflectometry, i.e. an increase of turbulence level from core towards edge, is reproduced. From $\rho_{\text {pol }}=0.5$ to 0.9 , both the measurement and the simulations show roughly an increase of a factor of five in $\tilde{n}$, which is a remarkable achievement. However, the GENE simulation with $P_{\mathrm{ECRH}}=1.8 \mathrm{MW}$ yields lower turbulence levels than the one with $P_{\mathrm{ECRH}}=0$, which is in contradiction to the measurement. Although for these simulations $\tilde{n}$ decreases with increased heating, $\tilde{T}_{i}$ and $\tilde{T}_{e}$ increase, which explains the increased heat fluxes and diffusivities from Fig. 17. It should be noted that in general the Doppler reflectometry measurement is linear with fluctuation amplitude only for low turbulence levels [93]. As the turbulence level increases, the Doppler reflectometry response eventually becomes non-linear and can even saturate [93]. It has been observed on many experiments that the radial turbulence profiles are low $(\tilde{n} / n<1 \%)$ in the core and several percent in the edge $[80,94]$. In agreement with these results, the radial turbulence level profile in Fig. 19 shows a radial increase of a factor of roughly 50 from the core to the edge. Hence, no saturation of the measurement is expected for the core measurement, while the edge data at around $\rho_{\text {pol }}=0.96$ could possibly be affected by saturation. Hence, saturation effects do not affect the comparative studies presented here, which focus specifically on the low-turbulence core part of the plasma.

\section{DISCUSSION}

Focusing on the case with $\rho_{\text {pol }}^{\mathrm{ECRH}}=0.5$, the main results obtained experimentally show the following picture: when ECRH is switched on, both $R / L_{T_{e}}$ and $R / L_{n}$ increase while $R / L_{T_{i}}$ decreases. Accompanying turbulence observations show that large-scale turbulence is most affected in that $u_{\perp}$ decreases (points more into the electron-diamagnetic direction) and $\tilde{n}$ increases, while smaller scales $\left(k_{\perp}>8 \mathrm{~cm}^{-1}\right)$ are unaffected. These results partly resemble measurements on trapped electron modes from DIII-D by DeBoo et al., who observed a predominant reaction of density turbulence at $k_{\theta} \approx 4-8 \mathrm{~cm}^{-1}$ in response to decreasing $R / L_{T_{e}}$ [6]. In their experiments, $\tilde{n}$ first decreased with decreasing $R / L_{T_{e}}$. As $R / L_{T_{e}}$ was further decreased, $\tilde{n}$ increased substantially. Similar experiments as in [6], also from DIII-D, were analyzed in substantial detail in various works [17, 95-97] investigating profile stiffness and responses of turbulence and heat fluxes to changes in $a / L_{T_{e}}$. These works found a critical gradient $a / L_{T_{e}}$, above which both heat fluxes and $T_{e}$ fluctuations in- 
creased $[95,96]$ and the propagation of turbulence changed towards the electron diamagnetic direction, visible in a change of Doppler spectral shape [97]. Also non-linear gyrokinetic simulations were compared with power balance results and $T_{e}$ flucutations [17]. In the present paper, the combined decrease of $R / L_{T_{i}}$ and increase of $R / L_{T_{e}}$ with additional ECRH at $\rho_{\text {pol }}>0.5$ brings the discharge closer to the transition region between ITG and TEM instabilites, defined here as the point where $v_{\text {ph }}$ changes sign. In contrast to the DIII-D experiments above, no transition into a clear TEM dominated regime is achieved. Both experiment and simulations point to a transition from a plasma in the ITG regime towards a situation in which ITG and TEM instabilities might co-exist [38], or at least the ITG character changes substantially.

Furthermore, the mixing length estimate states that $\tilde{n} / n \propto$ $1 /\left(k_{r} L_{n}\right)$, which can be written $\tilde{n} \propto \nabla n / k_{r}$. In the experiment it has been observed that for highest ECRH power at $\rho_{\text {pol }}^{\mathrm{ECRH}}=0.5, \nabla n$ at mid-radius increases substantially (cf Fig. 7), such that the increased $\tilde{n}$ is in accordance with this simple picture. Although no increase of $\tilde{n}$ at high $k_{\perp}$ is observed experimentally, the trend predicted by the mixing length estimate is at least recovered for small $k_{\perp}$.

In a broader context, the scales observed experimentally with Doppler reflectometry $\left(k_{\perp} \rho_{s}=0.7-4.2\right)$ are smaller than the scales where the growth rates peak in the gyrokinetic simulations $\left(k_{\perp} \rho_{s} \approx 0.4-0.7\right)$. This means that experimentally, the observations are at turbulence scales which are connected to the drive scale via an energy cascade. To be able to assess the question whether changes in the measured turbulence levels are due to changes in the drive or in the spectral energy transfer properties [85-87], detailed measurements of wavenumber spectra are necessary, which are not available for the discharges presented here.

The different $u_{\perp}$-reaction of large and small scales to strong mid-radius ECRH (cf Fig. 10(d)) is believed to be due to a change in phase velocity, since $v_{E \times B}$ is independent of $k_{\perp}$. In the experiment, a difference of approximately $3 \mathrm{~km} / \mathrm{s}$ is observed, while a simple assumption for the phase velocity of core turbulence is $v_{\mathrm{ph}} \approx 3 \rho_{s} c_{s} / R=1.6 \mathrm{~km} / \mathrm{s}[56,79]$. Both linear and non-linear gyrokinetic simulations show $v_{\text {ph }} \lesssim 500$ $\mathrm{m} / \mathrm{s}$, which is clearly below the measured value. An interpretation of the experimental observation of the different $u_{\perp}$ at different $k_{\perp}$ could be that, without ECRH, all scales propa-

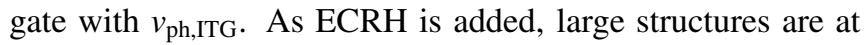
the transition to TEM (or in the TEM regime), such that the measured $v_{\text {ph }}$ includes contributions from ITG and TEM and a net $v_{\mathrm{ph}} \approx 0$ is measured. Unfortunately, with the present measurements, it is not possible to confirm or refute this speculation. Detailed core $E_{r}$ measurements, which will be possible thanks to an upgrade to the charge exchange recombination spectroscopy system [98], are necessary in order to calculate the absolute value of $v_{\mathrm{ph}}$.

\section{CONCLUSIONS}

Turbulence investigations comprising Doppler reflectometry, power balance analysis and non-linear gyrokinetic simula- tions have been presented for an H-mode discharge with four different ECRH power steps at two different radial locations in the AUG tokamak. Radial profiles of the perpendicular propagation velocity of density fluctuations and the density fluctuation level have been measured at different $k_{\perp}$ for all heating levels at both radial heating locations.

In general, the perpendicular velocity of density fluctuations in the core is NBI-driven and positive (measured at $\left.\rho_{\text {pol }}>0.5\right)$ and it exhibits a negative $u_{\perp}$-well in the edge region. Accompanying density turbulence level profiles show an increase from the core towards the edge, with a strong turbulence suppression close to the (strongly sheared) $u_{\perp}$ well. The effect of adding $P_{\mathrm{ECRH}}>1 \mathrm{MW}$ is a reduction of the large-scale core turbulence velocity with a concomitant increase in large-scale core turbulence level. The effects observed are consistent with a transition from a clearly ITG dominated plasma towards the TEM regime, either driven by the increased $R / L_{T_{e}}$ or $R / L_{n}$.

Linear gyrokinetic simulations show that the ITG is the fastest growing mode without ECRH. As ECRH is added, the changes in $R / L_{T_{i}}$ and $R / L_{T_{e}}$ change the character of the instability, resulting in a decrease of phase velocity. However, propagation is still in the ion-diamagnetic drift direction, indicating substantial ITG activity remains. Power balance analyses have been performed using the ASTRA code, the resulting heat fluxes have been used as a constraint for non-linear GENE simulations in the fluxtube limit. The heat fluxes can be reproduced if changes of $R / L_{T_{i}}$ used for GENE simulations of at most $20 \%$ are used. Although such a change is locally well within the error bars of the measurement, it is not if it has to be applied in a large fraction of the profile. More detailed studies are necessary in order to make a clear statement on that point. A central result worth pointing out is the agreement between experimental and gyrokinetic radial turbulence level profile, an investigation which has been done for the first time in AUG. Not only is the radial increase of $\tilde{n}$ reproduced qualitatively, also the relative increase from mid-radius towards the edge is reproduced quantitatively. Still, a discrepancy is found in the plasma response to additional ECRH heating: while the Doppler reflectometry turbulence level increases, the one from the gyrokinetic simulation slightly decreases. This will be investigated in further studies in which multi-dimensional parameter scans are to be included.

For the future, further experiments are planned to modify $R / L_{T_{e}}$ even more strongly to achieve a clear TEM dominated plasma. Furthermore, in-depth analysis of non-linear gyrokinetic simulations is planned in order to understand the discrepancy in response to additional heating power between experiment and simulation [87]. Apart from that, synthetic diagnostics employing a two-dimensional full-wave code will be developed to better understand the diagnostic response function [99]. Moreover, spectral power laws will be investigated in detail: experimental fine-resolution $k_{\perp}$ measurements are already at hand and will be compared with non-linear gyrokinetic simulations with applied synthetic diagnostics. These studies will provide more insight into the nature of the turbulence. 


\section{ACKNOWLEDGMENTS}

The authors would like to thank A. E. White and P. Hennequin for fruitful discussions. Furthermore the support from EFDA under work programs WP12-IPH-A05-1-06/PS-01/IPP, WP13-IPH-A05-P1-01/IPP, and fusion researcher fellowship WP12-FRF-IPP/Happel is gratefully acknowledged.
This work was partly performed in the framework of the Helmholtz Virtual Institute on plasma dynamical processes and turbulence using advanced microwave diagnostics. It is gratefully acknowledged that the results in this paper have been obtained with the assistance of high performance computing on the HELIOS system hosted at the International Fusion Energy Research Centre (IFERC) in Japan.
[1] E. Doyle, W. Houlberg, Y. Kamada, V. Mukhovatov, T. Osborne, A. Polevoi, G. Bateman, J. Connor, J. Cordey, T. Fujita, X. Garbet, T. Hahm, L. Horton, A. Hubbard, F. Imbeaux, F. Jenko, J. Kinsey, Y. Kishimoto, J. Li, T. Luce, Y. Martin, M. Ossipenko, V. Parail, A. Peeters, T. Rhodes, J. Rice, C. Roach, V. Rozhansky, F. Ryter, G. Saibene, R. Sartori, A. Sips, J. Snipes, M. Sugihara, E. Synakowski, H. Takenaga, T. Takizuka, K. Thomsen, M. Wade, H. Wilson, the ITPA Transport Physics Topical Group, the ITPA Confinement Database and Modelling Topical Group, and the ITPA Pedestal and Edge Topical Group, Nucl. Fusion 47, S18 (2007).

[2] F. Wagner, G. Becker, K. Behringer, D. Campbell, A. Eberhagen, W. Engelhardt, G. Fussmann, O. Gehre, J. Gernhardt, G. v. Gierke, G. Haas, M. Huang, F. Karger, M. Keilhacker, O. Klber, M. Kornherr, K. Lackner, G. Lisitano, G. G. Lister, H. M. Mayer, D. Meisel, E. R. Mller, H. Murmann, H. Niedermeyer, W. Poschenrieder, H. Rapp, and H. Röhr, Phys. Rev. Lett. 49, 1408 (1982).

[3] M. Shimada, D. Campbell, V. Mukhovatov, M. Fujiwara, N. Kirneva, K. Lackner, M. Nagami, V. Pustovitov, N. Uckan, J. Wesley, N. Asakura, A. Costley, A. Donné, E. Doyle, A. Fasoli, C. Gormezano, Y. Gribov, O. Gruber, T. Hender, W. Houlberg, S. Ide, Y. Kamada, A. Leonard, B. Lipschultz, A. Loarte, K. Miyamoto, V. Mukhovatov, T. Osborne, A. Polevoi, and A. Sips, Nucl. Fusion 47, S1 (2007).

[4] T. Rhodes, W. Peebles, M. V. Zeeland, D. Mikkelsen, M. Gilmore, X. Nguyen, D. Baker, J. DeBoo, J. deGrassie, W. Dorland, E. Doyle, C. Greenfield, C. Petty, and L. Zeng, Comparison of Broad Spectrum Turbulence Measurements and Gyrokinetic Code Predictions on the DIII-D Tokamak (Proc. 20 ${ }^{\text {th }}$ IAEA Fusion Energy Conference, Vilamoura, Portugal, 2004), EX/P6-23.

[5] T. L. Rhodes, W. A. Peebles, J. C. DeBoo, R. Prater, J. E. Kinsey, G. M. Staebler, J. Candy, M. E. Austin, R. V. Bravenec, K. H. Burrell, J. S. deGrassie, E. J. Doyle, P. Gohil, C. M. Greenfield, R. J. Groebner, J. Lohr, M. A. Makowski, X. V. Nguyen, C. C. Petty, W. M. Solomon, H. E. S. John, M. A. V. Zeeland, G. Wang, and L. Zeng, Plasma Phys. Control. Fusion 49, B183 (2007).

[6] J. C. DeBoo, C. Holland, T. L. Rhodes, L. Schmitz, G. Wang, A. E. White, M. E. Austin, E. J. Doyle, J. Hillesheim, W. A. Peebles, C. C. Petty, Z. Yan, and L. Zeng, Phys. Plasmas 17, 056105 (2010).

[7] T. L. Rhodes, C. Holland, S. P. Smith, A. E. White, K. H. Burrell, J. Candy, J. C. DeBoo, E. J. Doyle, J. C. Hillesheim, J. E. Kinsey, G. R. McKee, D. Mikkelsen, W. A. Peebles, C. C. Petty, R. Prater, S. Parker, Y. Chen, L. Schmitz, G. M. Staebler, R. E. Waltz, G. Wang, Z. Yan, and L. Zeng, Nucl. Fusion 51, 063022 (2011).

[8] F. Jenko, W. Dorland, M. Kotschenreuther, and B. N. Rogers,
Phys. Plasmas 7, 1904 (2000).

[9] M. Kotschenreuther, G. Rewoldt, and W. Tang, Comput. Phys. Commun. 88, 128 (1995).

[10] A. Peeters, Y. Camenen, F. Casson, W. Hornsby, A. Snodin, D. Strintzi, and G. Szepesi, Comput. Phys. Commun. 180, 2650 (2009), 40 \{YEARS\} \{OF\} CPC: A celebratory issue focused on quality software for high performance, grid and novel computing architectures.

[11] J. Candy and R. Waltz, J. Comput. Phys. 186, 545 (2003).

[12] V. Grandgirard, Y. Sarazin, X. Garbet, G. Dif-Pradalier, P. Ghendrih, N. Crouseilles, G. Latu, E. Sonnendrücker, N. Besse, and P. Bertrand, Commun. Nonlinear Sci. Numer. Simul. 13, 81 (2008).

[13] T.-H. Watanabe and H. Sugama, Nucl. Fusion 46, 24 (2006).

[14] M. Nunami, T.-H. Watanabe, and H. Sugama, Plasma Fus. Res. 5, 016 (2010).

[15] C. Holland, J. DeBoo, T. Rhodes, L. Schmitz, J. Hillesheim, G. Wang, A. White, M. Austin, E. Doyle, W. Peebles, C. Petty, L. Zeng, and J. Candy, Nucl. Fusion 52, 063028 (2012).

[16] C. Holland, C. Petty, L. Schmitz, K. Burrell, G. McKee, T. Rhodes, and J. Candy, Nucl. Fusion 52, 114007 (2012).

[17] C. Holland, J. E. Kinsey, J. C. DeBoo, K. H. Burrell, T. C. Luce, S. P. Smith, C. C. Petty, A. E. White, T. L. Rhodes, L. Schmitz, E. J. Doyle, J. C. Hillesheim, G. R. McKee, Z. Yan, G. Wang, L. Zeng, B. A. Grierson, A. Marinoni, P. Mantica, P. B. Snyder, R. E. Waltz, G. M. Staebler, and J. Candy, Nucl. Fusion 53, 083027 (2013).

[18] F. Jenko, D. Told, T. Görler, J. Citrin, A. Bañón Navarro, C. Bourdelle, S. Brunner, G. D. Conway, T. Dannert, H. Doerk, D. Hatch, J. Haverkort, J. Hobirk, G. Hogeweij, P. Mantica, M. Pueschel, O. Sauter, L. Villard, E. Wolfrum, and the ASDEX Upgrade Team, Nucl. Fusion 53, 073003 (2013).

[19] J. Citrin, F. Jenko, P. Mantica, D. Told, C. Bourdelle, J. Garcia, J. W. Haverkort, G. M. D. Hogeweij, T. Johnson, and M. J. Pueschel, Phys. Rev. Lett. 111, 155001 (2013).

[20] J. Citrin, F. Jenko, P. Mantica, D. Told, C. Bourdelle, R. Dumont, J. Garcia, J. Haverkort, G. Hogeweij, T. Johnson, M. Pueschel, and J.-E. contributors, Nucl. Fusion 54, 023008 (2014).

[21] D. R. Ernst, P. T. Bonoli, P. J. Catto, W. Dorland, C. L. Fiore, R. S. Granetz, M. Greenwald, A. E. Hubbard, M. Porkolab, M. H. Redi, J. E. Rice, K. Zhurovich, and the Alcator C-Mod Group, Phys. Plasmas 11, 2637 (2004).

[22] A. M. Dimits, G. Bateman, M. A. Beer, B. I. Cohen, W. Dorland, G. W. Hammett, C. Kim, J. E. Kinsey, M. Kotschenreuther, A. H. Kritz, L. L. Lao, J. Mandrekas, W. M. Nevins, S. E. Parker, A. J. Redd, D. E. Shumaker, R. Sydora, and J. Weiland, Phys. Plasmas 7, 969 (2000).

[23] W. M. Nevins, J. Candy, S. Cowley, T. Dannert, A. Dimits, W. Dorland, C. Estrada-Mila, G. W. Hammett, F. Jenko, M. J. Pueschel, and D. E. Shumaker, Phys. Plasmas 13, (2006). 
[24] W. M. Nevins, S. E. Parker, Y. Chen, J. Candy, A. Dimits, W. Dorland, G. W. Hammett, and F. Jenko, Phys. Plasmas 14, (2007).

[25] M. J. Pueschel, M. Kammerer, and F. Jenko, Phys. Plasmas 15, (2008).

[26] G. L. Falchetto, B. D. Scott, P. Angelino, A. Bottino, T. Dannert, V. Grandgirard, S. Janhunen, F. Jenko, S. Jolliet, A. Kendl, B. F. McMillan, V. Naulin, A. H. Nielsen, M. Ottaviani, A. G. Peeters, M. J. Pueschel, D. Reiser, T. T. Ribeiro, and M. Romanelli, Plasma Phys. Control. Fusion 50, 124015 (2008).

[27] R. V. Bravenec, J. Candy, M. Barnes, and C. Holland, Phys. Plasmas 18, (2011).

[28] A. E. White, L. Schmitz, G. R. McKee, C. Holland, W. A. Peebles, T. A. Carter, M. W. Shafer, M. E. Austin, K. H. Burrell, J. Candy, J. C. DeBoo, E. J. Doyle, M. A. Makowski, R. Prater, T. L. Rhodes, G. M. Staebler, G. R. Tynan, R. E. Waltz, and G. Wang, Phys. Plasmas 15, 056116 (2008).

[29] A. Casati, T. Gerbaud, P. Hennequin, C. Bourdelle, J. Candy, F. Clairet, X. Garbet, V. Grandgirard, O. D. Gürcan, S. Heuraux, G. T. Hoang, C. Honoré, F. Imbeaux, R. Sabot, Y. Sarazin, L. Vermare, and R. E. Waltz, Phys. Rev. Lett. 102, 165005 (2009).

[30] M. Greenwald, Phys. Plasmas 17, 058101 (2010).

[31] C. Holland, L. Schmitz, T. L. Rhodes, W. A. Peebles, J. C. Hillesheim, G. Wang, L. Zeng, E. J. Doyle, S. P. Smith, R. Prater, K. H. Burrell, J. Candy, R. E. Waltz, J. E. Kinsey, G. M. Staebler, J. C. DeBoo, C. C. Petty, G. R. McKee, Z. Yan, and A. E. White, Phys. Plasmas 18, 056113 (2011).

[32] S. Leerink, V. V. Bulanin, A. D. Gurchenko, E. Z. Gusakov, J. A. Heikkinen, S. J. Janhunen, S. I. Lashkul, A. B. Altukhov, L. A. Esipov, M. Y. Kantor, T. P. Kiviniemi, T. Korpilo, D. V. Kuprienko, and A. V. Petrov, Phys. Rev. Lett. 109, 165001 (2012).

[33] A. E. White, N. T. Howard, M. Greenwald, M. L. Reinke, C. Sung, S. Baek, M. Barnes, J. Candy, A. Dominguez, D. Ernst, C. Gao, A. E. Hubbard, J. W. Hughes, Y. Lin, D. Mikkelsen, F. Parra, M. Porkolab, J. E. Rice, J. Walk, S. J. Wukitch, and the Alcator C-Mod Team, Phys. Plasmas 20, 056106 (2013).

[34] T. Görler, A. E. White, D. Told, F. Jenko, C. Holland, and T. L. Rhodes, Phys. Plasmas 21, 122307 (2014).

[35] P. W. Terry, M. Greenwald, J.-N. Leboeuf, G. R. McKee, D. R. Mikkelsen, W. M. Nevins, D. E. Newman, D. P. Stotler, T. G. on Verification, Validation, U. B. P. Organization, and U. T. T. Force, Phys. Plasmas 15, (2008).

[36] M. Kammerer, F. Merz, and F. Jenko, Physics of Plasmas (1994present) 15, 052102 (2008).

[37] T. Görler and F. Jenko, Phys. Rev. Lett. 100, 185002 (2008).

[38] F. Merz and F. Jenko, Nucl. Fusion 50, 054005 (2010).

[39] M. A. Beer and G. W. Hammett, Phys. Plasmas 3, 4018 (1996).

[40] P. Malinov and F. Zonca, J. Plasma Phys. 71, 301 (2005).

[41] P. Hennequin, C. Honoré, A. Truc, A. Quéméneur, C. FenziBonizec, C. Bourdelle, X. Garbet, and G. T. Hoang, Nucl. Fusion 46, S771 (2006).

[42] T. Happel, T. Estrada, E. Blanco, C. Hidalgo, G. D. Conway, U. Stroth, and the TJ-II Team, Phys. Plasmas 18, 102302 (2011).

[43] L. Vermare, P. Hennequin, O. D. Gurcan, C. Bourdelle, F. Clairet, X. Garbet, R. Sabot, and the Tore Supra Team, Phys. Plasmas 18, 012306 (2011).

[44] L. Vermare, Ö. Gürcan, P. Hennequin, C. Honoré, X. Garbet, J. Giacalone, R. Sabot, and F. Clairet, C. R. Phys. 12, 115 (2011).

[45] T. Estrada, E. Ascasbar, E. Blanco, A. Cappa, P. H. Diamond, T. Happel, C. Hidalgo, M. Liniers, B. P. van Milligen, I. Pastor, D. Tafalla, and the TJ-II Team, Plasma Phys. Control. Fusion 54, 124024 (2012).

[46] F. Fernández-Marina, T. Estrada, and E. Blanco, Nucl. Fusion
54, 072001 (2014).

[47] M. Hirsch, E. Holzhauer, J. Baldzuhn, and B. Kurzan, Doppler Reflectometry for the Investigation of poloidally propagating Density Perturbations (Proc. $4^{\text {th }}$ International Reflectometry Workshop, Cadarache, France, 1999), report EUR-CEA-FC1674.

[48] X. L. Zou, T. F. Seak, M. Paume, J. M. Chareau, C. Bottereau, and G. Leclert, Poloidal Rotation Measurement in Tore Supra by Oblique Reflectometry (Proc. $4^{\text {th }}$ International Reflectometry Workshop, Cadarache, France, 1999), report EUR-CEA-FC1674.

[49] M. Hirsch, E. Holzhauer, J. Baldzuhn, B. Kurzan, and B. Scott, Plasma Phys. Control. Fusion 43, 1641 (2001).

[50] E. Poli, A. G. Peeters, and G. V. Pereverzev, Comput. Phys. Commun. 136, 90 (2001).

[51] G. D. Conway, E. Poli, T. Happel, and The ASDEX Upgrade Team, Plasma Fus. Res. 5, S2005 (2010).

[52] T. Estrada, T. Happel, L. Eliseev, D. López-Bruna, E. Ascasíbar, E. Blanco, L. Cupido, J. M. Fontdecaba, C. Hidalgo, R. Jiménez-Gómez, L. Krupnik, M. Liniers, M. E. Manso, K. J. McCarthy, F. Medina, A. Melnikov, B. v. Milligen, M. A. Ochando, I. Pastor, M. A. Pedrosa, F. L. Tabarés, D. Tafalla, and the TJ-II Team, Plasma Phys. Control. Fusion 51, 124015 (2009).

[53] E. Viezzer, T. Pütterich, G. D. Conway, R. Dux, T. Happel, J. C. Fuchs, R. M. McDermott, F. Ryter, B. Sieglin, W. Suttrop, M. Willensdorfer, E. Wolfrum, and the ASDEX Upgrade Team, Nucl. Fusion 53, 053005 (2013).

[54] H. W. Müller, J. Adamek, R. Cavazzana, G. D. Conway, J. P. Gunn, A. Herrmann, J. Horacek, C. Ionita, M. Kocan, M. Maraschek, C. Maszl, F. Mehlmann, B. Nold, M. Peterka, V. Rohde, R. Schrittwieser, N. Vianello, E. Wolfrum, M. Zuin, and the ASDEX Upgrade Team, Fluctuations, ELM Filaments and Turbulent Transport in the SOL at the Outer Midplane of ASDEX Upgrade (IAEA, Daejon, Korea, 2010), report EXD/P323.

[55] G. R. McKee, R. J. Fonck, D. K. Gupta, D. J. Schlossberg, M. W. Shafer, R. L. Boivin, and W. Solomon, Plasma Fus. Res. 2, S1025 (2007).

[56] G. D. Conway, C. Angioni, R. Dux, F. Ryter, A. G. Peeters, J. Schirmer, C. Troester, CFN Reflectometry Group, and the ASDEX Upgrade Team, Nucl. Fusion 46, S799 (2006).

[57] C. Tröster, Ph.D. thesis, Ludwig-Maximilians-Universität München, 2008.

[58] T. Happel, G. D. Conway, W. Kasparek, B. Plaum, C. Lechte, D. Wagner, U. Stroth, and the ASDEX Upgrade Team, Design of a new Doppler Reflectometer Front End for the ASDEX Upgrade Tokamak (Proc. 10 ${ }^{\text {th }}$ International Reflectometry Workshop, Padua, Italy, 2011).

[59] T. Happel, T. Estrada, E. Blanco, V. Tribaldos, A. Cappa, and A. Bustos, Rev. Sci. Instrum. 80, 073502 (2009).

[60] T. Happel, E. Blanco, and T. Estrada, Rev. Sci. Instrum. 81, 10D901 (2010).

[61] T. Happel, G. D. Conway, W. Kasparek, B. Plaum, U. Stroth, and the ASDEX Upgrade Team, Rev. Sci. Instrum. (2015), to be submitted.

[62] F. Ryter, G. Tardini, F. D. Luca, H.-U. Fahrbach, F. Imbeaux, A. Jacchia, K. Kirov, F. Leuterer, P. Mantica, A. Peeters, G. Pereverzev, W. Suttrop, and A. U. Team, Nucl. Fusion 43, 1396 (2003).

[63] A. G. Peeters, C. Angioni, M. Apostoliceanu, F. Jenko, F. Ryter, and the ASDEX Upgrade Team, Phys. Plasmas 12, 022505 (2005).

[64] B. Kurzan and H. D. Murmann, Rev. Sci. Instrum. 82, 103501 
(2011).

[65] M. Willensdorfer, E. Wolfrum, R. Fischer, J. Schweinzer, M. Sertoli, B. Sieglin, G. Veres, F. Aumayr, and the ASDEX Upgrade Team, Rev. Sci. Instrum. 83, 023501 (2012).

[66] E. Fable, C. Angioni, and O. Sauter, Plasma Phys. Control. Fusion 52, 015007 (2010).

[67] F. Jenko, W. Dorland, and G. W. Hammett, Phys. Plasmas 8, 4096 (2001).

[68] Y. Ren, S. M. Kaye, E. Mazzucato, W. Guttenfelder, R. E. Bell, C. W. Domier, B. P. LeBlanc, K. C. Lee, J. Luhmann, N. C., D. R. Smith, and H. Yuh, Phys. Rev. Lett. 106, 165005 (2011).

[69] T. Dannert and F. Jenko, Phys. Plasmas 12, 072309 (2005).

[70] C. Angioni, A. G. Peeters, F. Jenko, and T. Dannert, Phys. Plasmas 12, 112310 (2005).

[71] D. R. Ernst, J. Lang, W. M. Nevins, M. Hoffman, Y. Chen, W. Dorland, and S. Parker, Phys. Plasmas 16, 055906 (2009).

[72] R. M. McDermott, C. Angioni, R. Dux, E. Fable, T. Pütterich, F. Ryter, A. Salmi, T. Tala, G. Tardini, E. Viezzer, and the ASDEX Upgrade Team, Plasma Phys. Control. Fusion 53, 124013 (2011).

[73] R. M. McDermott, C. Angioni, R. Dux, A. Gude, T. Ptterich, F. Ryter, G. Tardini, and the ASDEX Upgrade Team, Plasma Phys. Control. Fusion 53, 035007 (2011).

[74] T. Happel, A. Bañón Navarro, G. D. Conway, C. Angioni, M. Bernert, M. Dunne, E. Fable, B. Geiger, T. Görler, F. Jenko, R. M. McDermott, F. Ryter, U. Stroth, and the ASDEX Upgrade Team, Wavenumber-resolved turbulence investigations in the ASDEX Upgrade tokamak and comparison to numerical simulations (Proc. 40 ${ }^{\text {th }}$ EPS Conference on Plasma Physics, Helsinki, Finland, 2013), ECA Vol. 37D, P1.188.

[75] K. H. Burrell, Phys. Plasmas 4, 1499 (1997).

[76] E. Viezzer, T. Pütterich, C. Angioni, A. Bergmann, R. Dux, E. Fable, R. McDermott, U. Stroth, E. Wolfrum, and the ASDEX Upgrade Team, Nucl. Fusion 54, 012003 (2014).

[77] T. Happel, G. D. Conway, P. Hennequin, C. Honoré, J.-C. Giacalone, P. Simon, U. Stroth, L. Vermare, and the ASDEX Upgrade Team, The optimized steerable W-band Doppler reflectometer on ASDEX Upgrade: possibilities and issues (Proc. $11^{\text {th }}$ International Reflectometry Workshop, Palaiseau, France, 2013).

[78] M. Hirsch, E. Holzhauer, J. Baldzuhn, and B. Kurzan, Rev. Sci. Instrum. 72, 324 (2001).

[79] M. A. Beer, Ph.D. thesis, 1995.

[80] P. C. Liewer, Nucl. Fusion 25, 543 (1985).

[81] L. Schmitz, L. Zeng, T. L. Rhodes, J. C. Hillesheim, E. J. Doyle, R. J. Groebner, W. A. Peebles, K. H. Burrell, and G. Wang, Phys. Rev. Lett. 108, 155002 (2012).

[82] K. Ida, S. Hidekuma, Y. Miura, T. Fujita, M. Mori, K. Hoshino, N. Suzuki, T. Yamauchi, and the JFT-2M Group, Phys. Rev. Lett. 65, 1364 (1990).

[83] R. J. Groebner, K. H. Burrell, and R. P. Seraydarian, Phys. Rev.
Lett. 64, 3015 (1990).

[84] K. H. Burrell, T. N. Carlstrom, E. J. Doyle, D. Finkenthal, P. Gohil, R. J. Groebner, D. L. Hillis, J. Kim, H. Matsumoto, R. A. Moyer, T. H. Osborne, C. L. Rettig, W. A. Peebles, T. L. Rhodes, H. StJohn, R. D. Stambaugh, M. R. Wade, and J. G. Watkins, Plasma Phys. Control. Fusion 34, 1859 (1992).

[85] V. Bratanov, F. Jenko, D. R. Hatch, and M. Wilczek, Phys. Rev. Lett. 111, 075001 (2013).

[86] A. Bañón Navarro, B. Teaca, F. Jenko, G. W. Hammett, T. Happel, and A. U. Team, Phys. Plasmas 21, (2014).

[87] A. Bañón Navarro, T. Happel, T. Görler, F. Jenko, J. Abiteboul, A. Bustos, H. Doerk, D. Told, and the ASDEX Upgrade Team, Phys. Plasmas (2015), submitted.

[88] G. Pereverzev and P. N. Yushmanov, Technical Report No. IPP 5/98, Max-Planck Institute (unpublished).

[89] P. Xanthopoulos, W. A. Cooper, F. Jenko, Y. Turkin, A. Runov, and J. Geiger, Phys. Plasmas 16, 082303 (2009).

[90] H. Doerk and et al., Phys. Plasmas (2015), to be submitted.

[91] N. Howard, M. Greenwald, D. Mikkelsen, M. Reinke, A. White, D. Ernst, Y. Podpaly, and J. Candy, Nucl. Fusion 52, 063002 (2012).

[92] N. T. Howard, C. Holland, A. E. White, M. Greenwald, and J. Candy, Physics of Plasmas (1994-present) 21, 112510 (2014).

[93] E. Blanco and T. Estrada, Plasma Phys. Control. Fusion 50, 095011 (2008).

[94] R. Sabot, F. Clairet, G. D. Conway, L. Cupido, X. Garbet, G. Falchetto, T. Gerbaud, S. Hacquin, P. Hennequin, S. Heuraux, C. Honoré, G. Leclert, L. Meneses, A. Sirinelli, L. Vermare, and A. Truc, Plasma Phys. Control. Fusion 48, B421 (2006).

[95] J. C. DeBoo, C. C. Petty, A. E. White, K. H. Burrell, E. J. Doyle, J. C. Hillesheim, C. Holland, G. R. McKee, T. L. Rhodes, L. Schmitz, S. P. Smith, G. Wang, and L. Zeng, Phys. Plasmas 19, 082518 (2012).

[96] J. C. Hillesheim, J. C. DeBoo, W. A. Peebles, T. A. Carter, G. Wang, T. L. Rhodes, L. Schmitz, G. R. McKee, Z. Yan, G. M. Staebler, K. H. Burrell, E. J. Doyle, C. Holland, C. C. Petty, S. P. Smith, A. E. White, and L. Zeng, Phys. Rev. Lett. 110, 045003 (2013).

[97] J. C. Hillesheim, J. C. DeBoo, W. A. Peebles, T. A. Carter, G. Wang, T. L. Rhodes, L. Schmitz, G. R. McKee, Z. Yan, G. M. Staebler, K. H. Burrell, E. J. Doyle, C. Holland, C. C. Petty, S. P. Smith, A. E. White, and L. Zeng, Phys. Plasmas 20, 056115 (2013).

[98] R. M. McDermott and et al., Rev. Sci. Instrum. (2015), to be submitted.

[99] C. Lechte, G. D. Conway, T. Görler, C. Tröster, and the ASDEX Upgrade Team, Doppler Reflectometry Simulations for AS$D E X$ Upgrade (Proc. 41 ${ }^{\text {th }}$ EPS Conference on Plasma Physics, Berlin, Germany, 2014), ECA Vol. 38F, O3.115. 